\title{
Joint Altitude and Hybrid Beamspace Precoding Optimization for UAV-enabled Multiuser mmWave MIMO System
}

\author{
Zhen Chen, Member, IEEE, Nan Zhao, Senior Member, IEEE, Daniel Ka Chun So, Senior Member, IEEE, \\ Jie Tang, Senior Member, IEEE, Boyi Tang, Xiu Yin Zhang, Fellow, IEEE, and Kai-Kit Wong, Fellow, IEEE
}

\begin{abstract}
The combination of unmanned aerial vehicles (UAVs) and millimeter wave (mmWave) multiple-input multipleout (MIMO) system is regarded as a key enabling technology for beyond 5G networks, as it provides high data rate aerial links. However, establishing UAV-enabled mmWave MIMO communication is quite challenging due to the high hardware cost in terms of radio frequency (RF) chains. As a cost-effective alternative, a beamspace precoding with discrete lens arrays (DLA) architecture has received considerable attention. However, the underlying optimal design in beamspace precoding has not been fully exploited in UAV-enabled communication scenario. In this paper, the joint design of the UAV's altitude and hybrid beamspace precoding is proposed for the UAV-enabled multiuser MIMO system, in which the DLA is exploited to reduce the number of the $\mathrm{RF}$ chain. In the proposed scheme, the optimization problem is formulated as a minimum weighted mean squared error (MWMSE) method. Then an efficient algorithm with the penalty dual decomposition (PDD) is proposed that aims to jointly optimize the altitude of UAV, beam selection and digital precoding matrices. Simulation results confirm the comparable performance of the proposed scheme and perform close to fulldigital beamforming in terms of achievable spectral efficiency.
\end{abstract}

Index Terms-UAV, mmWave communication, MIMO, hybrid

This work has been supported in part by Key Research and Development Project of Guangdong Province under Grant 2019B010156003, 2021A1515011966, in part by National Key Research and Development Project under Grant 2019YFB1804100, in part by the National Natural Science Foundation of China under Grant 61971194, 62001171, in part by the Natural Science Foundation of Guangdong Province under Grant 2019A1515011607, in part by the Open Research Fund of National Mobile Communications Research Laboratory, Southeast University (No. 2019D06), in part by Guangxi technology basement and talent program under Grant 2020AC15010, and in part by the Research Fund Program of Guangdong Key Laboratory of Aerospace Communication and Networking Technology under Grant 2018B030322004. (Corresponding author:Jie Tang)

Z. Chen and X. Zhang are with the School of Electronic and Information Engineering, South China University of Technology, Guangzhou, China. (email:chenz@scut.edu.cn and eexyz@scut.edu.cn).

N. Zhao is with the School of Information and Communication Engineering, Dalian University of Technology, Dalian, China. (e-mail: zhaonan@dlut.edu.cn).

B. Tang is with the School of Commerce, University of Electronic Science and Technology of China, Chengdu, China. (e-mail: tangboyi_2000@163.com).

D. K. C. So is with the School of Electrical and Electronic Engineering, University of Manchester, Manchester, United Kingdom. (e-mail: d.so@manchester.ac.uk).

J. Tang is with the School of Electronic and Information Engineering, South China University of Technology, Guangzhou, China, and also with the National Mobile Communications Research Laboratory, Southeast University, Nanjing 210096, China (e-mail: eejtang@ scut.edu.cn).

K.-K Wong is with the Department of Electronic and Electrical Engineering, University College London, London, United Kingdom. (e-mail: kaikit.wong@ucl.ac.uk). beamspace precoding, lens antenna arrays.

\section{INTRODUCTION}

$\mathbf{U}$ NMANNED aerial vehicles (UAVs) have recently proven to be of immense help in both military and civilian fields and can be endowed with multiple-input-multiple-output (MIMO) for wireless communication networks. Different from terrestrial communications, UAVs can provide an ondemand flexible platform for deploying aerial base station (BS) to support temporary or urgent events, which enhances the wireless capacity for ground terminals (GTs) [1], [2]. Particularly, UAVs are used to provide various services, such as sampling data from dangerous areas, firefighting and disaster rescue [3]. In addition, UAVs are envisioned to be a prime candidates of future mmWave communication systems [4]. Because of the short wavelength at mmWave frequency, massive antenna arrays can be deployed on a UAV to form the beam-steerable directive beam, which is witnessed as a promising approach to offer available data rates and extensive coverage [5], [6]. However, the desired beamforming gains in conventional UAVenabled system tend to rely on the fully digital precoding structure, where one radio frequency (RF) chain is required to serve one antenna [7]. An increase in RF chain has resulted in high hardware cost, which obstruct the commercial deployment of UAV-enabled mmWave MIMO systems [8]. It has been proven that RF chains may consume up to $70 \%$ of the total transceiver power [7], [9]-[11]. Therefore, high hardware cost (RF chain) makes the UAV-enabled mmWave MIMO system unrealistic to low-cost communications.

To tackle this challenge, lots of studies have been developed to reduce the energy consumption and the number of RF chains, such as load-controlled parasitic antenna arrays (LCPAAs) [12], [13], beam selection [14]-[17] and hybrid ana$\log$ /digital precoding design [18]-[22]. The recent concept of "beamspace MIMO" has been exploited as a potential scheme to substantiality reduce the hardware cost of RF chains, where the discrete lens array (DLA) is considered for analog spatial beamforming [23], [24]. In a beamspace MIMO systems, the electromagnetic (EM) lens with the antenna array are developed for analog spatial beamforming domain [23]. To compensate the high attenuation of mmWave frequencies, the different directions of mmWave signals can be focused on the antenna array, which is referred to as beamspace channel [25]. Due to the high path loss at mmWave frequencies, the 

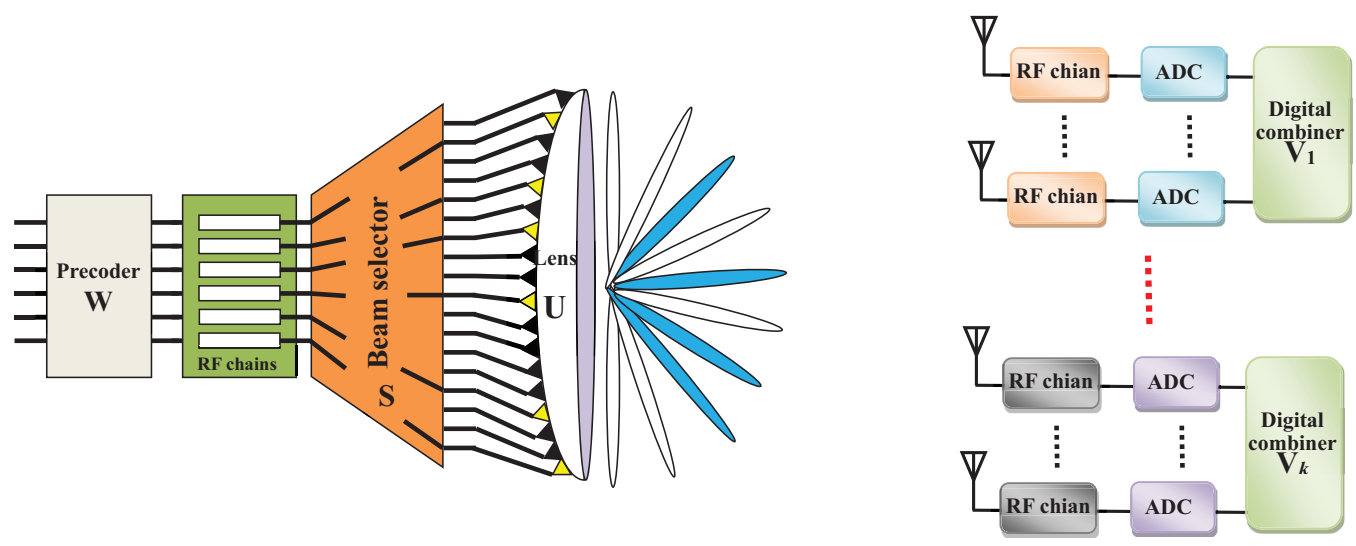

Fig. 1. Illustration of the mmWave MIMO communication with the LAAs.

beamspace mmWave channel with lens antenna array (LAA) is sparse, meaning that only a part of beams carry the majority of the information [26]. Thus the beam selector is designed to determine the energy-focusing beams, which can reduce the required number of RF chains with only a negligible performance degradation [27], [28]. However, the beam selection is not a trivial task. The traditional beam selection problem is maximum magnitude (the works in "MM-BS") so as to obtain a higher transmission rate. Subsequently, [29] designs an interference-aware precoding strategy for multiuser interference system, which is simpler and less expensive than the MM-BS schemes. However, these works are restricted to the terrestrial communications, where the beam selection techniques are developed for mmWave MIMO systems primarily considered a static or quasi-static scenario. It is commonly believed that the mmWave MIMO systems networks with fixed infrastructure are vulnerable to unforeseen or temporary events (e.g., natural disasters, sports events). Therefore, it is important to develop the UAV-enabled beamspace precoding schemes to deal with emergencies promptly.

\section{A. Related Works and Motivation}

There are a recent surge of studies on the use of UAVs for mmWave communications [5], [30]-[34]. To assess the applicability of UAV deployment in mmWave system, it is critical to design a comprehensive and efficient hybrid beamforming (HBF), while also taking into account the effects of mmWave high attenuation propagation. However, one major drawback of the hybrid analog/digital precoding is the requirement of a large number of RF chains and phase shifters, which lead to extra power consumption and hardware cost [22]. It should be noted that the hybrid precoding/combining matrices optimization problem is more difficult and complicated than the traditional fully digital one [33]-[35]. One effective and commonly used approach is to exploit the energy-focusing capability of lens, such hybrid analog/digital transceiver architecture is designed as shown in Fig. 1. The key idea is to exploit the DLA for mmWave MIMO communications. In [24], a path division multiplexing (PDM) paradigm was introduced in DLA-based mmWave MIMO system, where parallel data streams were transmitted over different propagation paths. In [36], the full-dimensional DLA-based mmWave system was developed in multi-user mmWave scenarios using a path division multiple access strategy. In [37], the highdimensional DLA for the beamspace MIMO system was investigated and was then extended to the high-dimensional multiuser communication scenarios [38]. In beamspace MIMO system, beam selection schemes often are studied to significantly reduce the RF complexity while obtaining near-optimal performances [7]. However, the same beam at the BS is likely selected for different users, which may cause inter-user interference in the beamspace MIMO system. To tackle this problem, an interference-aware scheme for beam selection was investigated in [29]. Subsequently, the compressed channel estimation was studied in mmWave beamspace MIMO system by exploiting the structure of LAAs [39]. It is worth noting that the beamspace MIMO system is essentially similar to the phase shifting and selection in dynamic hybrid precoding system [40], but the beamspace MIMO uses analog to digital converters (ADCs) to significantly lower hardware cost and alleviate the performance bottleneck caused by the multiuser interference. Therefore, applying DLA to UAV-enabled wireless communication will have tremendous potential.

However, the hybrid beamspace precoding optimization scheme for UAV-enabled mmWave MIMO communication is still an open problem. The primary concern for UAV-enabled mmWave MIMO system is that the altitude of UAV and the beam selector with LAAs will make the problem highly nonconvex and difficult to solve. In addition, the joint optimizing transmit precoder and receive combiners often are coupled with each other. Most of the existing works focus on simple decoupling mechanisms, in which the original optimization problem is divided into transmit and receive precoding subproblems, and then focus on the constant modulus constraint in solving the subproblems. These techniques are effective but follow a heuristic design way. Moreover, such decoupling mechanisms may result in a performance loss in some cases, since a fixed digital precoder is usually not optimal. In addition, these prior works are restricted to terrestrial communications 
whose results do not consider the presence of UAVs. This motivates our work to propose more efficient techniques for UAV-enabled mmWave communications.

\section{B. Main Contributions}

The integration of UAV and beamspace MIMO is viewed as a promising technique in the upcoming mmWave communication, and hence has drawn great interests recently. Most of the prior art on UAV-enabled mmWave MIMO system [30]-[34] does not consider the LAAs to substantially reduce the RF chains and the power consumption. In particular, these known approaches are restricted to phase shifters whose results cannot be extended directly to UAV-enabled mmWave beamspace MIMO systems. In addition, the works in [29], [36]-[38], [40] proposed the joint optimizing transmit and receive beamforming scheme for mmWave beamspace MIMO system that minimize mean squared error (MSE) or maximize the sumrate. However, these works do not consider the presence of UAV. Especially, in practical UAV-enabled communication scenarios, the random movement of the UAV will result in the appearance and disappearance of scatterers, which lead to a considerable performance loss. Therefore, jointly optimizing the altitude of UAV and hybrid beamspace precoding in UAVenabled mmWave MIMO communication is a problem worth investigating and exploring. Motivated by the above studies, this work proposes the joint optimization of altitude of UAV, the beam selection and beamspace precoding for UAV-enabled mmWave MIMO system. The contributions in this article can be summarized as follows.

- Firstly, an efficient joint optimization approach is proposed for UAV-enabled mmWave beamspace MIMO system. Apart from the above mentioned challenges in the existing joint optimization problem, the altitude of UAV, the digital precoder/combiner variables, the location planning and altitude of UAV are also considered in the UAV-enabled mmWave scenario.

- The proposed optimization problem is NP-hard, which makes it difficult to achieve acceptable results. To tackle this problem, the penalty dual decomposition (PDD) method is considered by introducing a series of equality constraints with auxiliary variables and penalty hyperparameters to cope with the coupling digital precoding/combining matrices and beam selector constraints. After that the optimization problem is reformulated as equivalent augmented Lagrangian (AL) function, and seeks its minimizer via an AL method, where each subproblem is solved independently by using the block coordinate descent (BCD) algorithm.

- To achieve the optimal flight altitude of UAV, a lowcomplexity scheme is proposed, where the two cases are distinguished by checking whether the given altitude is sufficient for the UAV to serve its associated GTs. Numerical results validate the effectiveness of the proposed strategy of jointly optimizing the altitude of UAV, beam selection and the beamspace precoding, and achieve a satisfactory performance close to that of the fully digital precoder.

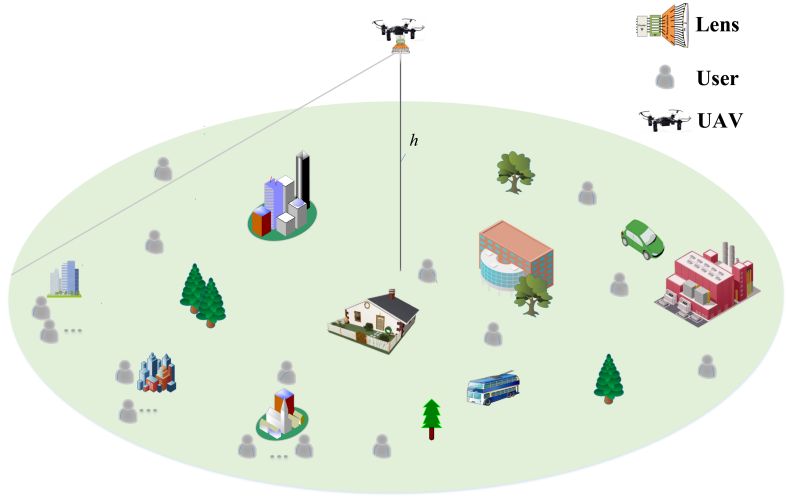

Fig. 2. Illustration of a UAV-enabled hybrid beamspace beamforming for multiuser mmWave communication systems.

\section{Organization and Notation}

The remain of this paper is organized as follows. The Section II presents the system model of a multi-user mmWave beamspace system along with the joint optimization problem formulation. In Section III, we present the basic idea and the optimization procedure. In Section IV, the simulation results are provided to validate the theoretical findings, followed by the conclusions in Section V.

Notations: Scalar is denoted by lower-case letters; lowerand upper-case boldface letters are used for vectors and matrices, respectively; $\operatorname{tr}(\cdot)$ is the trace of a matrix; $\log (\cdot)$ is used for the logarithm; $I_{K}$ denotes the $K \times K$ identity matrix; The superscripts $(\cdot)^{T},(\cdot)^{H}$, denote the transpose and conjugate transpose, respectively; $\mathfrak{R e}\{\mathbf{x}\}$ and $\mathbf{x}^{H}$ are the real part and conjugate of a complex signal $\mathbf{x}$, respectively; $(\cdot)_{j}$ is denoted by the $j$-th column of a matrix; $(\cdot)_{i, j}$ denotes $i, j$-th element of a matrix; $\mathcal{C N}\left(m, \sigma^{2}\right)$ represents the symmetric complexvalued Gaussian distributions with mean $m$ and covariance $\sigma^{2} ;\|\cdot\|_{2},\|\cdot\|_{F}, \operatorname{diag}(\cdot)$ and $\mathbb{E}[\cdot]$ denote the Euclidean norm, Frobenius norm, diagonalization and expectation operators, respectively.

\section{PROBLEM FORMULATION}

We describe a downlink mmWave beamspace communication, where a UAV is deployed as a flying base-station (BS) to serve $K$ simultaneous GTs in Fig.2. The UAV is equipped with LAAs. We assume that the UAV with horizontal and vertical position $\mathbf{x}=(x(1), x(2))$ is hovering at altitude of $h$ meters above the ground level. The height of each GT is assumed to be zero compared with the height of the UAV. $\mathbf{z}_{k}=\left(z_{k}(1), z_{k}(2)\right)$ is used for the location of GT $k$. Thus, the ground coverage area of the UAV depend on the antenna's main lobe with radius $\left\|\mathbf{z}-\mathbf{x}_{k}\right\|_{2}=h \mathbf{t a n} \Theta$. The UAV with $N_{t}$ transmit antennas and $N_{t}^{R F}$ chains serves a total of $K$ GTs each of which is equipped with $N_{r}$ receive antennas. It is assumed that $N_{s}$ data streams are transmitted to a receiver, which are subject to constraints $K N_{s} \leq N_{t}^{R F}<N_{t}$ and $N_{s} \leq N_{r}$.

Due to the sparsity and high free-space path loss at mmWave band, we consider the Rician fading channel model [41]. Different from the conventional low frequency channels, the 
mmWave channels between UAV and GTs are stochastic fading, and thus the channel matrix from the $k$-th GT to the UAV can be expressed as [42]

$$
\hat{\boldsymbol{H}}_{k}=\sqrt{\beta_{0}\left(d_{k}\right)^{-\alpha}}\left(\sqrt{\frac{\delta}{\delta+1}} G_{k}+\sqrt{\frac{1}{\delta+1}} \hat{\boldsymbol{G}}_{k}\right),
$$

where $\beta_{0}$ is the channel power gain, $d_{k}=\sqrt{\left\|\mathbf{z}-\mathbf{x}_{k}\right\|_{2}^{2}+h^{2}}$ is the distance between the UAV and GT $k$ and $\alpha \geq 2$ denotes the path loss exponent. $G_{k}$ is the LoS component of the GT $k$ with $G_{k}(i, j)=1, \hat{G}_{k}$ denotes the the Rayleigh fading channel (or NLoS) component of the GT $k$ and $\delta \geq 0$ is the Rician factor specifying the power ratio between the dominant LoS and NLoS components.

\section{A. The Lens-aided mmWave MIMO System}

As shown in Fig. 1, the lens antenna arrays are designed on the UAV-enabled mmWave MIMO system. In this structure, the signals arriving from different unknown directions will be focused on the array antennas. Such the lens $\boldsymbol{U}$ can be characterized as a spatial discrete fourier transform of the incident signals, which includes the array steering vectors of $N_{t}$ covering the entire angular domain as follows [43]

$$
\boldsymbol{U}=\left[\mathbf{a}\left(\bar{\phi}_{1}\right), \mathbf{a}\left(\bar{\phi}_{2}\right), \ldots, \mathbf{a}\left(\bar{\phi}_{N_{t}}\right)\right]^{H},
$$

where $\mathbf{a}\left(\bar{\phi}_{i}\right)=\frac{1}{\sqrt{N_{t}}}\left[e^{-2 j \pi \bar{\phi}_{i} n}\right]_{n \in \mathcal{I}}$ is the $N_{t} \times 1$ array steering vector for the spatial direction $\bar{\phi}_{i}$, and $\mathcal{I}=\left\{n-\left(N_{t}-\right.\right.$ 1) $\left./ 2 \mid n=0,1, \ldots, N_{t}-1\right\}$ is an index set of array elements. $\bar{\phi}_{i}$ is the normalized spatial directions [21]

$$
\bar{\phi}_{i}=\frac{1}{N_{t}}\left(i-\frac{N_{t}-1}{2}\right), \quad i=1,2, \ldots, N_{t} .
$$

Thus, the physical spatial MIMO channel $\hat{\boldsymbol{H}}_{k}$ in (1) can be obtained with the Fourier transformation, which is defined as [24]

$$
\tilde{\boldsymbol{H}}_{k}=\hat{\boldsymbol{H}}_{k} \boldsymbol{U}=\left(d_{k}\right)^{-\frac{\alpha}{2}} \boldsymbol{H}_{k}
$$

where $\boldsymbol{H}_{k}=\sqrt{\frac{\beta_{0} \delta}{\delta+1}} \boldsymbol{G}_{k} \boldsymbol{U}+\sqrt{\frac{\beta_{0}}{\delta+1}} \hat{\boldsymbol{G}}_{k} \boldsymbol{U}$.

For each GT, the received signal is processed with a digital combiner $\boldsymbol{V}_{k} \in \mathbb{C}^{N_{r} \times N_{s}}$. Then, the received signal $\mathbf{y}_{k}$ at the $k$-th GT is formulated as

$\mathbf{y}_{k}=\boldsymbol{V}_{k}^{H} \tilde{\boldsymbol{H}}_{k} \boldsymbol{S} \boldsymbol{W} \boldsymbol{c}+\boldsymbol{V}_{k}^{H} \boldsymbol{n}_{k}=\left(d_{k}\right)^{-\frac{\alpha}{2}} \boldsymbol{V}_{k}^{H} \boldsymbol{H}_{k} \boldsymbol{S} \boldsymbol{W} \boldsymbol{c}+\boldsymbol{V}_{k}^{H} \boldsymbol{n}_{k}$

where $\boldsymbol{W}=\left[\boldsymbol{W}_{1}, \boldsymbol{W}_{2}, \ldots, \boldsymbol{W}_{K}\right] \in \mathbb{C}^{N_{t}^{R F} \times K N_{s}}$ is the digital precoder and $\boldsymbol{W}_{k} \in \mathbb{C}^{N_{t}^{R F} \times N_{s}}, 1 \leq k \leq K$ denotes the digital precoder for the $k$-th GT, $\boldsymbol{c}=\left[\boldsymbol{c}_{1}^{T}, \boldsymbol{c}_{2}^{T}, \ldots, \boldsymbol{c}_{K}^{T}\right]^{T} \in \mathbb{C}^{K N_{s} \times 1}$ denotes the transmitted signal vector for $K$ GTs with normalized power $\mathbb{E}\left(\boldsymbol{c} \boldsymbol{c}^{T}\right)=\boldsymbol{I} . \boldsymbol{n}_{k} \sim \mathcal{C N}\left(0, \sigma^{2} \boldsymbol{I}_{k}\right)$ is additive white Gaussian noise (AWGN) and the element $s_{i, j}$ of beam selection $\boldsymbol{S} \in \mathbb{C}^{N_{t} \times N_{t}^{R F}}$ is set to either 0 or 1 . It implies that each column of beam selector has one and only one non-zero element " 1 ".

\section{B. Joint Optimization Problem Formulation}

Before proceeding with the detailed derivation analysis, an equivalent $\left(d_{k}\right)^{-\frac{\alpha}{2}}$ is reformulated to make a model mathematically more tractable, which can be rewritten as $\left(d_{k}\right)^{-\frac{\alpha}{2}}=$ $\left(h^{2}+\left\|\mathbf{z}-\mathbf{x}_{k}\right\|_{2}^{2}\right)^{-\frac{\alpha}{2}}=\left(h^{2}+(h \tan \Theta)^{2}\right)^{-\frac{\alpha}{2}}=(h \sec \Theta)^{-\alpha}$. Thus, the received signal of GT $k$ can be simplified as

$$
\mathbf{y}_{k}=(h \sec \Theta)^{-\alpha} \boldsymbol{V}_{k}^{H} \boldsymbol{H}_{k} \boldsymbol{S} \boldsymbol{W} \boldsymbol{c}+\boldsymbol{V}_{k}^{H} \boldsymbol{n}_{k} .
$$

Under the independence assumption of $\left\{\boldsymbol{c}_{k}, \boldsymbol{n}_{k} \mid k=\right.$ $1, \ldots, K\}$, we can obtain the well-known MMSE combining matrix $V_{k}$ by minimizing the MMSE, which is formulated as

$$
\begin{aligned}
\boldsymbol{V}_{k}=(h \sec \Theta)^{\alpha}\left(\boldsymbol{H}_{k} \boldsymbol{S} \boldsymbol{W} \boldsymbol{W}^{H} \boldsymbol{S}^{H} \boldsymbol{H}_{k}^{H}\right. \\
\left.+\sigma^{2}(h \sec \Theta)^{2 \alpha} \boldsymbol{I}_{N_{r}}\right)^{-1} \boldsymbol{H}_{k} \boldsymbol{S} \boldsymbol{W} .
\end{aligned}
$$

According to (6), the hybrid precoders optimization problem is designed by assuming the combining matrix is used in (7). Thus, the proposed joint optimization problem is to maximize the spectral efficiency (SE) of the downlink system, which is formulated as

$$
\begin{aligned}
& \max _{\boldsymbol{W}, \boldsymbol{S}, h} \sum_{k=1}^{K} \log \operatorname{det}\left(\boldsymbol{I}_{N_{r}}+\frac{\boldsymbol{H}_{k} \boldsymbol{S} \boldsymbol{W} \boldsymbol{W}^{H} \boldsymbol{S}^{H} \boldsymbol{H}_{k}^{H}}{\sigma^{2}(h \mathbf{s e c} \Theta)^{2 \alpha}}\right) \\
& \text { s.t. } \operatorname{tr}\left(\boldsymbol{W}^{H} \boldsymbol{S}^{H} \boldsymbol{S} \boldsymbol{W}\right) \leq P, \\
& \quad \sum_{i=1}^{N_{t}} s_{i, j}=1, \quad \sum_{j=1}^{N_{t}^{R F}} s_{i, j} \leq 1, \quad s_{i, j} \in\{0,1\}, \\
& h_{\min } \leq h \leq h_{\max }, \\
& (7),
\end{aligned}
$$

where $P$ is the total transmit power at the BS. Due to the effect of obstacle heights and authority regulations of UAVs, the feasible region of height $h$ is determined as $\left[h_{\min }, h_{\max }\right]$. The constraints $\sum_{j=1}^{N_{r}^{R F}} s_{i, j} \leq 1, i=1,2, \ldots, N_{t}$ guarantee that each beam is selected for at most one RF chain, while the constraints $\sum_{i=1}^{N_{t}} s_{i, j}=1, j=1,2, \ldots, N_{r}^{R F}$ ensure that each beam is generated by a single RF chain.

It is worth mentioning that the optimization problem (8) involves joint optimization over three variables along with nonconvex constraints, which is difficult to solve straightforwardly. Furthermore, the fixed combining matrix would lead to the performance reducing. To solve above issues, the equivalent optimization problem is proposed that transforms the problem (8) into a more tractable form. Firstly, MSE is introduced as the objective function for the joint altitude of UAV, the beam selection and transmit/receive precoding design, which can be expressed as $\boldsymbol{E}_{k}=\mathbb{E}\left\{\left(\mathbf{y}_{k}-\boldsymbol{c}_{k}\right)\left(\mathbf{y}_{k}-\boldsymbol{c}_{k}\right)^{H}\right\}$ [44]. Moreover, since $\mathbf{y}_{k}$ and $\boldsymbol{n}_{k}$ are mutually independent, $\boldsymbol{E}_{k}$ can be further expressed as

$$
\begin{aligned}
\boldsymbol{E}_{k}=( & (h \sec \Theta)^{-2 \alpha} \boldsymbol{V}_{k}^{H} \boldsymbol{H}_{k} \boldsymbol{S} \boldsymbol{W} \boldsymbol{W}^{H} \boldsymbol{S}^{H} \boldsymbol{H}_{k}^{H} \boldsymbol{V}_{k} \\
& -2(h \sec \Theta)^{-\alpha} \mathfrak{R e}\left(\boldsymbol{V}_{k}^{H} \boldsymbol{H}_{k} \boldsymbol{S} \boldsymbol{W}\right)+\sigma^{2} \boldsymbol{V}_{k}^{H} \boldsymbol{V}_{k}+\boldsymbol{I}_{N_{s}} .
\end{aligned}
$$


By introducing auxiliary weighting matrices $\xi_{k}$, the sum-rate maximization problem (8) is equivalently expressed as follows

$$
\begin{aligned}
& \min _{\boldsymbol{W}, \boldsymbol{V}_{k}^{H}, \boldsymbol{S}, h, \xi_{k}} \sum_{k=1}^{K}\left(\log \operatorname{det}\left(\xi_{k}\right)-\operatorname{tr}\left(\xi_{k} \boldsymbol{E}_{k}\right)+N_{s}\right) \\
& \text { s.t. } \quad \operatorname{tr}\left(\boldsymbol{W}^{H} \boldsymbol{S}^{H} \boldsymbol{S} \boldsymbol{W}\right) \leq P, \\
& \quad \sum_{j=1}^{N_{t}^{R F}} s_{i, j} \leq 1, \quad \sum_{i=1}^{N_{t}} s_{i, j}=1, \quad s_{i, j} \in\{0,1\}, \\
& h_{\min } \leq h \leq h_{\max },
\end{aligned}
$$

where $\xi_{k}$ is obtained by $\boldsymbol{E}_{k}^{-1}$.

Proof: The proof is given in Appendix.

Instead of the original optimization problem (8), a more tractable minimum weighted mean squared error (MWMSE) optimization problem (10) is considered. For this kind of combinatorial optimization problem, the optimal digital combiner for each GT are separable, and can be estimated independently. Therefore, the optimization problem (10) can be decomposed as the subproblems $\boldsymbol{V}_{k}^{H}=\arg \min _{\boldsymbol{V}_{k}^{H}} \boldsymbol{E}_{k}$ for $k=1,2, \ldots, K$. Considering the partial derivative of (10a) with respect to $\boldsymbol{V}_{k}^{H}$ and ignoring the constant term $\xi_{k}$, we have

$$
\boldsymbol{V}_{k}^{H}=\boldsymbol{W}_{k}^{H} \overline{\boldsymbol{H}}_{k}^{H}\left(\overline{\boldsymbol{H}}_{k} \boldsymbol{W} \boldsymbol{W}^{H} \overline{\boldsymbol{H}}_{k}^{H}+\sigma^{2} \boldsymbol{I}_{N_{r}}\right)^{-1},
$$

where $\overline{\boldsymbol{H}}_{k}=(h \sec \Theta)^{-\alpha} \boldsymbol{H}_{k} \boldsymbol{S}$.

For the digital precoder $\boldsymbol{W}$ at the UAV, the MSE of $K$ GTs is considered, i.e., $\boldsymbol{E}=\sum_{k=1}^{K} \boldsymbol{E}_{k}$. Similar to (9), $\boldsymbol{E}$ can be expressed as

$$
\begin{aligned}
\boldsymbol{E}= & \operatorname{tr}\left(\boldsymbol{V}^{H} \overline{\boldsymbol{H}} \boldsymbol{W} \boldsymbol{W}^{H} \overline{\boldsymbol{H}}^{H} \boldsymbol{V}\right)-\operatorname{tr}\left(\boldsymbol{V}^{H} \overline{\boldsymbol{H}} \boldsymbol{W}\right) \\
& +\sigma^{2} \operatorname{tr}\left(\boldsymbol{V}^{H} \boldsymbol{V}\right)-\operatorname{tr}\left(\boldsymbol{W}^{H} \overline{\boldsymbol{H}}^{H} \boldsymbol{V}\right)+K N_{s} .
\end{aligned}
$$

where $\overline{\boldsymbol{H}}=\left[\overline{\boldsymbol{H}}_{1}, \overline{\boldsymbol{H}}_{2}, \ldots, \overline{\boldsymbol{H}}_{K}\right]^{T}, \boldsymbol{V}=\operatorname{diag}\left(\boldsymbol{V}_{1}, \boldsymbol{V}_{2}, \ldots, \boldsymbol{V}_{K}\right)$ represents the digital combiner for $K$ GTs. According to the Karush-Kuhn-Tucker (KKT) condition, the optimal solution of $\boldsymbol{W}$ can be solved by the following closed form

$$
\boldsymbol{W}=\left(\overline{\boldsymbol{H}}^{H} \boldsymbol{V} \boldsymbol{V}^{H} \overline{\boldsymbol{H}}\right)^{-1} \overline{\boldsymbol{H}}^{H} \boldsymbol{V} .
$$

From (11) and (13), it can observe that the solutions of $\boldsymbol{V}_{k}^{H}$ and $\boldsymbol{W}$ depend on each other. To solve this optimization problem, the classical BCD algorithm is usually used to divide the MWMSE optimization problem into a sequence of subproblems, and optimize each variable separately. However, when different variables in problem (10) are coupling constraints for each other, the BCD algorithm cannot be directly performed. To tackle the coupling constraint, the penalty function method will be introduced to solve the minimization problem (10). Next, the BCD algorithm with penalty function is proposed to solve the each of subproblems.

\section{Proposed Hybrid Precoding Design}

The main efforts of this section is solve the optimization problem (10) by exploiting BCD algorithm based on the augmented Lagrangian method. Note that the transmit precoder and receive combiners are coupled with each other in the power constraint, which is difficult to achieve the optimal solution. To this end, the original problem is divided into a series of sub-problems: weighting matrices $\xi_{k}$, altitude of UAV $h$, digital combiner $\boldsymbol{V}_{k}^{H}$, digital precoder $\boldsymbol{W}$, beam selector $\boldsymbol{S}$ and solving each independently. The specific procedures are summarized as follows.

\section{A. Optimization of the Weighting Matrices $\xi_{k}$}

Under fixed variables $\boldsymbol{W}, \boldsymbol{V}_{k}^{H}, \boldsymbol{S}$ and $h$, the optimization problem (10) with respect to $\xi_{k}$ is rewritten as

$$
\min _{\xi_{k}} \sum_{k=1}^{K} \log \operatorname{det}\left(\xi_{k}\right)-\operatorname{tr}\left(\xi_{k} \boldsymbol{E}_{k}\right) \text {. }
$$

By taking the derivative of the optimization problem (14) with respect to $\xi_{k}$ to zero. Thus, the optimal $\xi_{k}^{*}$ can be obtained as

$$
\begin{aligned}
\xi_{k}^{*}= & \boldsymbol{E}_{k}^{-1}=\left[(h \sec \Theta)^{-2 \alpha} \boldsymbol{V}_{k}^{H} \boldsymbol{H}_{k} \boldsymbol{S} \boldsymbol{W} \boldsymbol{W}^{H} \boldsymbol{S}^{H} \boldsymbol{H}_{k}^{H} \boldsymbol{V}_{k}\right. \\
& \left.-2(h \sec \Theta)^{-\alpha} \mathfrak{R e}\left(\boldsymbol{V}_{k}^{H} \boldsymbol{H}_{k} \boldsymbol{S} \boldsymbol{W}\right)+\sigma^{2} \boldsymbol{V}_{k}^{H} \boldsymbol{V}_{k}+\boldsymbol{I}_{N_{s}}\right]^{-1} .
\end{aligned}
$$

\section{B. Optimization of the Altitude $h$}

We investigate the altitude planning of UAV with fixed $\xi_{k}$, $\boldsymbol{W}, \boldsymbol{V}_{k}^{H}$ and $\boldsymbol{S}$. Then, the optimization problem (10) with respect to $h$ is formulated as

$$
\begin{aligned}
& \min _{h} \sum_{k=1}^{K}(h \sec \Theta)^{-2 \alpha} A_{k}-(h \sec \Theta)^{-\alpha} B_{k}+C_{k}, \\
& \text { s.t. } \quad h_{\min } \leq h \leq h_{\max },
\end{aligned}
$$

where $A_{k}=\operatorname{tr}\left(\xi_{k} \boldsymbol{V}_{k}^{H} \boldsymbol{H}_{k} \boldsymbol{S} \boldsymbol{W} \boldsymbol{W}^{H} \boldsymbol{S}^{H} \boldsymbol{H}_{k}^{H} \boldsymbol{V}_{k}\right), \quad B_{k}=$ $2 \operatorname{tr}\left(\boldsymbol{V}_{k}^{H} \boldsymbol{H}_{k} \boldsymbol{S} \boldsymbol{W}\right), \boldsymbol{C}_{k}=\left[\sigma^{2} \operatorname{tr}\left(\xi_{k} \boldsymbol{V}_{k}^{H} \boldsymbol{V}_{k}\right)+\xi_{k} \boldsymbol{I}_{N_{s}}\right]$.

Based on (16), defining function $f(\boldsymbol{x}(h)) \triangleq A_{k} \boldsymbol{x}^{2}(h)-$ $B_{k} \boldsymbol{x}(h)+C_{k}$ and $\boldsymbol{x}(h) \triangleq(h \sec \Theta)^{-\alpha}$, we have

$$
f^{\prime}(\boldsymbol{x}(h))=2 A_{k} \boldsymbol{x}(h)-B_{k} \text {. }
$$

It follows that the optimal altitude of UAV is obtained in the following two cases.

1) Case 1: If $\boldsymbol{x}(h)>\frac{B_{k}}{2 A_{k}}$, we have $f^{\prime}(\boldsymbol{x}(h))>0$, and $f(\boldsymbol{x}(h))$ is an increasing function when $\hat{h}_{\min } \leq h \leq h_{\max }$. The optimal value of $h$ is denoted by $h^{*}$. Then, it is observed that the $\boldsymbol{x}(h)$ is a monotonic decreasing function with respect to $h$, which yields

$$
h^{*}=h_{\max } .
$$

2) Case 2: If $\boldsymbol{x}(h) \leq \frac{B_{k}}{2 A_{k}}$ and $\hat{h}_{\min } \leq h \leq h_{\max }, f(\boldsymbol{x}(h))$ is a monotonically decreasing function. The optimal $\boldsymbol{x}(h)^{*}$ is

$$
h^{*}=h_{\min } .
$$

After the flight altitude $h^{*}$ is obtained, a 2D exhaustive search is used to achieve the optimal location planning $\mathbf{x}$ of UAV to the optimization problem (16). Symmetries are an abvious device to cut out portions of the configuration space, which can reduce the size of the search space by a factor of about $1 / 8$ [45]. In the revised manuscript, 2D exhaustive search with symmetries scheme is considered to reduce the complexity from $\mathcal{O}\left(2^{K}\right)$ to $\mathcal{O}\left(1.85^{K}\right)$. 
C. Optimization of the Beam Selection and Beamspace Precoding Matrix

To tackle the coupling constraint, the framework of the classic penalty dual decomposition (PDD) [46] is adopted to uncouple the problem (10). Before proceeding to the derivation of the PDD method, the auxiliary variables $\left\{\hat{s}_{i, j}\right\}$ and $\boldsymbol{Q}_{k}$ are introduced, which are subject to the constraints of $s_{i, j}=\hat{s}_{i, j}$, $s_{i, j}\left(1-\hat{s}_{i, j}\right)=0,0 \leq \hat{s}_{i, j} \leq 1$ and $\boldsymbol{Q}_{k}=\boldsymbol{S} \boldsymbol{W}_{k}$. Let $\boldsymbol{s}_{i}^{T} \in \mathbb{C}^{1 \times N_{t}^{R F}}$ be the $i$-th row of $\boldsymbol{S}$, the constraint $\sum_{j=1}^{N_{r}^{R^{T}}} s_{i, j} \leq 1$ is redefined as $\boldsymbol{s}_{i}^{T} \mathbf{1} \leq 1$ and the elements of vector $\mathbf{1} \in \mathbb{C}^{N_{t}^{R F} \times 1}$ are 1 . Furthermore, $s_{i, j}$ is represented as $\boldsymbol{s}_{i}^{T} \boldsymbol{f}_{j}$, where $\boldsymbol{f}_{j} \in \mathbb{C}^{N_{r}^{R F} \times 1}$ is the $j$-th column of $\boldsymbol{I}_{N_{r}^{R F}}$. According to above notations, the optimization problem (10) is reformulated as follows

$$
\begin{aligned}
& \min _{\boldsymbol{W}_{k}, \boldsymbol{V}_{k}^{H}, \boldsymbol{Q}_{k},\left\{\hat{s}_{i, j}\right\}} \sum_{k=1}^{K} \operatorname{tr}\left(\xi_{k} \boldsymbol{E}_{k}\right) \\
& \text { s.t. } \sum_{k=1}^{K}\left\|\boldsymbol{Q}_{k}\right\|_{F}^{2} \leq P, \\
& \boldsymbol{Q}_{k}=\boldsymbol{S} \boldsymbol{W}_{k}, \\
& \boldsymbol{s}_{i}^{T} \boldsymbol{f}_{j}\left(1-\hat{s}_{i, j}\right)=0, \quad \boldsymbol{s}_{i}^{T} \boldsymbol{f}_{j}-\hat{s}_{i, j}=0, \\
& \sum_{i=1}^{N_{t}} \boldsymbol{s}_{i}^{T} \boldsymbol{f}_{j}=1, \\
& \boldsymbol{s}_{i}^{T} \mathbf{1} \leq 1, \quad 0 \leq \hat{s}_{i, j} \leq 1,
\end{aligned}
$$

where $\boldsymbol{E}_{k}$ is given by

$$
\boldsymbol{E}_{k}=\left(\boldsymbol{I}-\boldsymbol{V}_{k}^{H} \boldsymbol{H}_{k} \boldsymbol{Q}_{k}\right)\left(\boldsymbol{I}-\boldsymbol{V}_{k}^{H} \boldsymbol{H}_{k} \boldsymbol{Q}_{k}\right)^{H}+\sigma^{2} \boldsymbol{V}_{k}^{H} \boldsymbol{V}_{k} .
$$

It turns out that the minimization problem (20) is still not easy to solve. To tackle these difficulties, the BCD algorithm is proposed to obtain the suboptimal solution. For this purpose, the above constraint minimization problem is transformed into an unconstrained one by adopting the penalty function. The multiplier variables $\left\{\boldsymbol{L}_{k}\right\}$ is introduced for the constraints $\left\{\boldsymbol{Q}_{k}=\boldsymbol{S} \boldsymbol{W}_{k}\right\}$ and the problem (20) is given by

$$
\begin{aligned}
\boldsymbol{W}_{k}, \boldsymbol{V}_{k}^{H}, \boldsymbol{Q}_{k},\left\{\hat{s}_{i, j}\right\}, s_{j} & \sum_{k=1}^{K} \operatorname{tr}\left(\xi_{k} \boldsymbol{E}_{k}\right) \\
+ & \frac{1}{2 \rho} \sum_{k=1}^{K}\left\|\boldsymbol{Q}_{k}-\boldsymbol{S} \boldsymbol{W}_{k}+\rho \boldsymbol{L}_{k}\right\|_{F}^{2} \\
+ & \frac{1}{2 \rho} \sum_{j=1}^{N_{t}^{R F}}\left(\sum_{i=1}^{N_{t}} \boldsymbol{s}_{i}^{T} \boldsymbol{f}_{j}-1+\rho \mu_{j}\right)^{2} \\
+ & \frac{1}{2 \rho} \sum_{i=1}^{N_{t}} \sum_{j=1}^{N_{t}^{R F}}\left(\boldsymbol{s}_{i}^{T} \boldsymbol{f}_{j}\left(1-\hat{s}_{i, j}\right)+\left(\boldsymbol{s}_{i}^{T} \boldsymbol{f}_{j}-\hat{s}_{i, j}\right)+\rho \lambda_{i, j}\right)^{2}, \\
\text { s.t. } & \sum_{k=1}^{K}\left\|\boldsymbol{Q}_{k}\right\|_{F}^{2} \leq P, \\
& \boldsymbol{s}_{i}^{T} \mathbf{1} \leq 1, \quad 0 \leq \hat{s}_{i, j} \leq 1,
\end{aligned}
$$

where $\rho$ is the scalar penalty parameter, $\lambda_{i, j}$ and $\mu_{j}$ are Lagrangian multipliers corresponding to the minimum rate constraints.
In the following, we focus on solving the corresponding optimization problem (21) by exploiting BCD type algorithms. The optimization variables are divided into a number of blocks variables $\boldsymbol{W}_{k}, \boldsymbol{V}_{k}^{H}, \boldsymbol{Q}_{k}$ and $\boldsymbol{S}$, such that for each block the corresponding subproblem can be solved independently. Specifically, the details about the process of each subproblems are given as follows.

1) The Subproblem $\left\{\boldsymbol{V}_{k}^{H}\right\}$ : We optimize the variables $\boldsymbol{V}_{k}^{H}$ with fixing the other variables. The $\boldsymbol{V}_{k}^{H}$ is optimized through the following problem

$$
\min _{\boldsymbol{V}_{k}^{H}} \sum_{k=1}^{K} \operatorname{tr}\left(\xi_{k} \boldsymbol{E}_{k}\right) .
$$

And then, using the KKT condition of (22) with respect to $\boldsymbol{V}_{k}^{H}$, the optimal $\boldsymbol{V}_{k}$ can be derived as a function of $\boldsymbol{Q}_{k}$ as follows

$\boldsymbol{V}_{k}^{H}=(h \mathbf{s e c} \Theta)^{\alpha} \boldsymbol{Q}_{k}^{H} \boldsymbol{H}_{k}^{H}\left(\boldsymbol{H}_{k} \boldsymbol{Q}_{k} \boldsymbol{Q}_{k}^{H} \boldsymbol{H}_{k}^{H}+\sigma^{2}(h \mathbf{s e c} \Theta)^{2 \alpha} \boldsymbol{I}\right)^{-1}$.

2) The Subproblem w.r.t. $\boldsymbol{W}_{k}$ : The optimized $\boldsymbol{W}_{k}$ is achieved by solving the following minimization problem

$$
\min _{\boldsymbol{W}_{k}}\left\|\boldsymbol{Q}_{k}-\boldsymbol{S} \boldsymbol{W}_{k}+\rho \boldsymbol{L}_{k}\right\|_{F}^{2} .
$$

Similarly, the optimal $\boldsymbol{W}_{k}$ can be obtained by exploiting the optimality conditions of first order in (24), which yields

$$
\boldsymbol{W}_{k}=\left(\boldsymbol{S}^{H} \boldsymbol{S}\right)^{-1} \boldsymbol{S}^{H}\left(\boldsymbol{Q}_{k}-\rho \boldsymbol{L}_{k}\right) .
$$

3) The Subproblem w.r.t. $\left\{\boldsymbol{Q}_{k}\right\}$ : The subproblems of optimizing $\left\{\boldsymbol{Q}_{k}\right\}$ are updated by solving the following minimization problem

$$
\begin{gathered}
\min _{\boldsymbol{Q}_{k}} \sum_{k=1}^{K}\left\{\operatorname{tr}\left(\xi_{k} \boldsymbol{E}_{k}\right)+\frac{1}{\rho}\left\|\boldsymbol{Q}_{k}-\boldsymbol{S} \boldsymbol{W}_{k}+\rho \boldsymbol{L}_{k}\right\|_{F}^{2}\right\} \\
\text { s.t. } \quad \sum_{k=1}^{K}\left\|\boldsymbol{Q}_{k}\right\|_{F}^{2} \leq P .
\end{gathered}
$$

By expanding $\left\|\boldsymbol{Q}_{k}-\boldsymbol{S} \boldsymbol{W}_{k}+\rho \boldsymbol{L}_{k}\right\|_{F}^{2}$ and some appropriate rearrangement, the minimization problem (26) can be equivalently rewritten as

$$
\begin{aligned}
\min _{\boldsymbol{Q}_{k}} \sum_{k=1}^{K}\left\{\operatorname{tr}\left(\xi_{k} \boldsymbol{E}_{k}\right)+\frac{1}{\rho} \operatorname{tr}\left(\boldsymbol{Q}_{k}^{H} \boldsymbol{Q}_{k}\right)-\frac{2}{\rho} \mathfrak{R e}\left\{\operatorname{tr}\left(\boldsymbol{Q}_{k}^{H} \boldsymbol{B}_{k}\right)\right\}\right\} \\
\text { s.t. } \quad \sum_{k=1}^{K}\left\|\boldsymbol{Q}_{k}\right\|_{F}^{2} \leq P
\end{aligned}
$$

where $\boldsymbol{B}_{k}$ is introduced for simplicity and given by $\boldsymbol{B}_{k}=$ $\left(\boldsymbol{S} \boldsymbol{W}_{k}-\rho \boldsymbol{L}_{k}\right)$.

The above problem (27) is a convex quadratic optimization problem, which can be solved successively according to the Lagrangian function. By attaching a Lagrange multiplier $\eta \geq$ 0, the closed-form solution of the optimal $\boldsymbol{Q}_{k}$ is given by

$\boldsymbol{Q}_{k}=\left(\boldsymbol{I}+\rho \eta \boldsymbol{I}+\rho \boldsymbol{H}_{k}^{H} \boldsymbol{V}_{k} \xi_{k} \boldsymbol{V}_{k}^{H} \boldsymbol{H}_{k}\right)^{-1}\left(\boldsymbol{B}_{k}+2 \rho \boldsymbol{H}_{k}^{H} \boldsymbol{V}_{k} \xi_{k}\right)$. 
where $\eta$ is chosen by using bisection method.

4) The Subproblem w.r.t. $\left\{\hat{s}_{i, j}\right\}$ : The variable $\left\{\hat{s}_{i, j}\right\}$ is updated by fixing the remaining variables. The minimization subproblem with respect to $\left\{\hat{s}_{i, j}\right\}$ can be rewritten as

$$
\begin{aligned}
& \min _{\hat{s}_{i, j}} \frac{1}{2 \rho}\left(\boldsymbol{s}_{i}^{T} \boldsymbol{f}_{j}\left(1-\hat{s}_{i, j}\right)+\left(\boldsymbol{s}_{i}^{T} \boldsymbol{f}_{j}-\hat{s}_{i, j}\right)+\rho \lambda_{i, j}\right)^{2} \\
& \text { s.t. } \quad 0 \leq \hat{s}_{i, j} \leq 1 .
\end{aligned}
$$

It is not difficult to find that the problem (29a) is a scalar continuously differentiable function. By enforcing the KKT condition, the optimization problem (29a) can be solved by

$$
\hat{s}_{i, j}=\frac{2 s_{i, j}^{2}+\left(2+\rho \lambda_{i, j}\right) s_{i, j}+\rho \lambda_{i, j}}{\left(1+s_{i, j}\right)^{2}} .
$$

Recalling that $\hat{s}_{i, j}$ satisfies $0 \leq \hat{s}_{i, j} \leq 1$, the solution of the constrained problem (29) is easily given by

$$
s_{i, j}^{*}=\left\{\begin{array}{cl}
1, & 1 \leq \hat{s}_{i, j}, \\
\hat{s}_{i, j}, & 0<\hat{s}_{i, j}<1, \\
0, & \hat{s}_{i, j} \leq 0 .
\end{array}\right.
$$

5) The Subproblem w.r.t. $\left\{\boldsymbol{s}_{i}\right\}$ : The subproblem of optimizing $\left\{\boldsymbol{s}_{i}\right\}$ can be updated by introducing the Lagrange multiplier, which yields

$$
\begin{aligned}
\min _{\boldsymbol{s}_{i}} & \frac{1}{2 \rho} \sum_{j=1}^{N_{t}^{R F}}\left(\sum_{i=1}^{N_{t}} \boldsymbol{s}_{i}^{T} \boldsymbol{f}_{j}-1+\rho \mu_{j}\right)^{2} \\
& +\frac{1}{2 \rho} \sum_{i=1}^{N_{t}} \sum_{j=1}^{N_{t}^{R F}}\left(\boldsymbol{s}_{i}^{T} \boldsymbol{f}_{j}\left(1-\hat{s}_{i, j}\right)+\left(\boldsymbol{s}_{i}^{T} \boldsymbol{f}_{j}-\hat{s}_{i, j}\right)+\rho \lambda_{i, j}\right)^{2} \\
& \text { s.t. } \quad \boldsymbol{s}_{i}^{T} \mathbf{1} \leq 1, i=1,2, \ldots, N_{t} .
\end{aligned}
$$

It is clear that the optimization problem (32) associated with each $\boldsymbol{s}_{i}$ are convex. By examining the KKT optimality condition, the closed-form solution of optimization problem (32) with respect to $s_{i}$ yields:

$$
\begin{aligned}
\boldsymbol{s}_{i} & =\left(3 \boldsymbol{I}+\sum_{j=1}^{N_{t}^{R F}}\left(\hat{s}_{i, j}^{2}-2 \hat{s}_{i, j}\right) \boldsymbol{f}_{j} \boldsymbol{f}_{j}^{T}\right)^{-1} \\
& \times\left(\sum_{j=1}^{N_{t}^{R F}}\left(\rho \lambda_{i, j}\left(1-\hat{s}_{i, j}\right)+\left(\sum_{k=i}^{N_{t}} \boldsymbol{s}_{k}^{T} \boldsymbol{f}_{j}+\rho \mu_{j}-1\right)\right) \boldsymbol{f}_{j}\right) .
\end{aligned}
$$

Finally, the beam selector $S$ can be solved by using the oneiteration BCD method, which is summarized in Algorithm 1. The remaining task is to update $\left\{\boldsymbol{L}_{k}\right\},\left\{\lambda_{i, j}\right\},\left\{\mu_{j}\right\}$ and $\rho$. The multiplier variables $\left\{\boldsymbol{L}_{k}\right\},\left\{\lambda_{i, j}\right\}$ and $\left\{\mu_{j}\right\}$ are updated as follows

$$
\begin{aligned}
& \boldsymbol{L}_{k}^{(n+1)}=\boldsymbol{L}_{k}^{(n)}+\frac{1}{\rho^{(n)}}\left(\boldsymbol{Q}_{k}-\boldsymbol{S} \boldsymbol{W}_{k}\right), \\
& \lambda_{i, j}^{(n+1)}=\lambda_{i, j}^{(n)}+\frac{1}{\rho^{(n)}}\left(\boldsymbol{s}_{i}^{T} \boldsymbol{f}_{j}\left(2-\hat{s}_{i, j}\right)-\hat{s}_{i, j}\right), \\
& \mu_{j}^{(n+1)}=\mu_{j}^{(n)}+\frac{1}{\rho^{(n)}}\left(\sum_{i=1}^{N_{t}}\left(\boldsymbol{s}_{i}^{T} \mathbf{1}-1\right)\right)
\end{aligned}
$$

and the penalty parameter $\rho$ is updated as follows

$$
\rho^{(n+1)}=\left\{\begin{array}{cc}
\kappa \rho^{(n)}, & \frac{\left\|\boldsymbol{E}_{k}^{(n)}-\boldsymbol{E}_{k}^{(n-1)}\right\|_{F}^{2}}{\left\|\boldsymbol{E}_{k}^{(n-1)}-\boldsymbol{E}_{k}^{(n-2)}\right\|_{F}^{2}}>\varepsilon, \\
\rho^{(n)}, & \text { otherwise, }
\end{array}\right.
$$

where $\kappa>0, \varepsilon<1$ are used to control the penalty parameter $\rho$. The detailed description for solving the optimization problem (10) is provided in Algorithm 2.
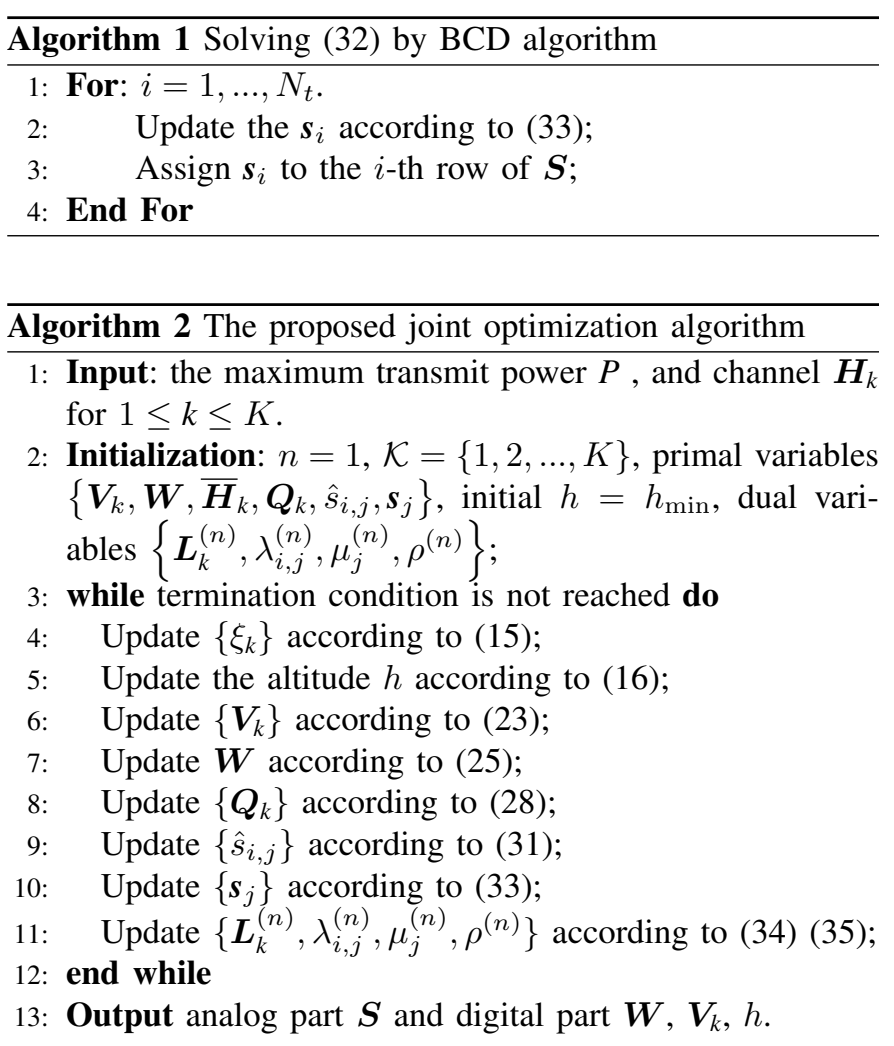

We estimate the computational complexity of the proposed Algorithm 2 by computing the number of required multiplications. The main computational complexity of Algorithm 2 is caused by solving the augmented Lagrange problem (10) which consists of four sub-problems, i.e., (14), (16), (22), (24), (27), (29) and (32). The complexities of solving (14) is $\mathcal{O}\left(N_{s}^{3}+N_{r} N_{s}^{2}\right)$. The complexities of solving (16) is $\mathcal{O}\left(N_{s}^{3}+1.85^{K}\right)$. The complexities of solving (22), (24), (27) and (29) are $\mathcal{O}\left(K N_{r} N_{s} N_{t}^{R F}+N_{r}^{3}\right)$, $\mathcal{O}\left(\left(N_{t}^{R F}\right)^{2} N_{t}+\left(N_{t}^{R F}\right)^{3}+K N_{t} N_{s} N_{t}^{R F}\right), \mathcal{O}\left(\left(N_{t}^{R F}\right)^{3}+\right.$ $\left.\left(N_{t}^{R F}\right)^{2} N_{t}+N_{t}^{R F} N_{s} N_{t}\right)$ and $\mathcal{O}\left(N_{t} N_{t}^{R F}\right)$. The complexities of solving (32) is $\mathcal{O}\left(N_{t}\left(\left(N_{t}^{R F}\right)^{2}+\left(N_{t}^{R F}\right)^{3}\right)\right)$. Therefore, the overall complexity of Algorithm 2 is $\mathcal{O}\left(I\left(N_{s}^{3}+N_{r} N_{s}^{2}+\right.\right.$ $1.85^{K}+N_{r}^{2}+\left(N_{t}^{R F}\right)^{2} N_{t}+N_{t}\left(N_{t}^{R F}\right)^{3}+K N_{t} N_{s} N_{t}^{R F}+$ $\left.K N_{t} N_{t}^{R F}\right)$ ), where $I$ is the numbers of iterations.

Finally, the issue of the convergence of Algorithm 2 is considered. The main convergence result is provided in Theorem 1.

Theorem 1. Let $\left\{h, \boldsymbol{V}_{k}, \boldsymbol{W}, \overline{\boldsymbol{H}}_{k}, \hat{\boldsymbol{s}}_{i, j}, \boldsymbol{Q}_{k}, \boldsymbol{s}_{j},\right\}$ be the sequence generated by Algorithm 2. If the Robinson's condition holds for the optimization problem (10). Then, there exists one 


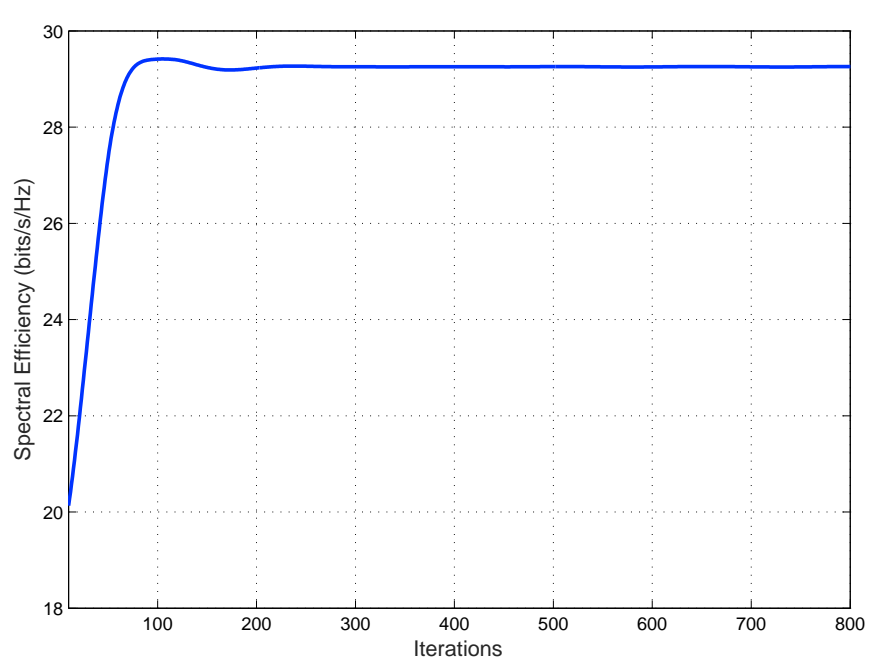

Fig. 3. Achievable SE comparison versus the the number of iterations.

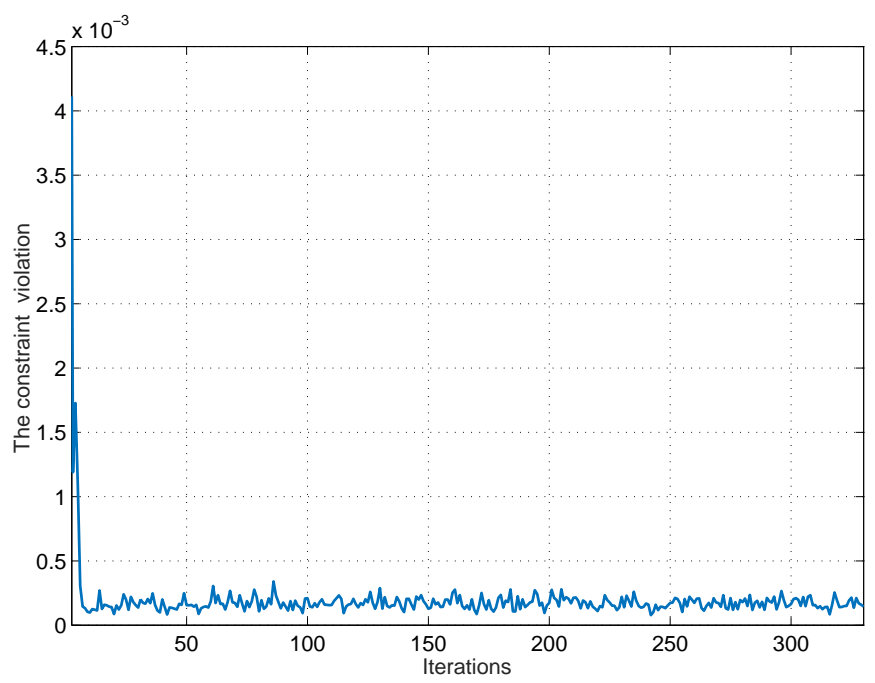

Fig. 4. The constraint violation comparison versus the the number of iterations.

limit point of sequence of iterations generated by the $B C D$ algorithm, which is a stationary point for the optimization problem (10).

Proof: The detailed proof process of convergence for a general algorithmic framework was provided in [47], [48].

\section{Simulation Results}

In this section, simulation results are provided to analyse the effectiveness of the proposed UAV-enabled mmWave MIMO system with the lens antenna arrays. We investigate the SE performance of the proposed UAV-enabled beamspace MIMO scheme and make comparisons with several recent works. Unless specified otherwise, we assume that the UAV is equipped with a DLA to serve $K=4$ GTs. The antenna number of $\mathrm{UAV}$ is $N_{t}=64$ and the number of RF chains is $N_{t}^{R F}=12$. The channel power gain with the reference distance $d_{0}=1 \mathrm{~m}$ is $\beta_{0}=-40 \mathrm{~dB}$. The GTs are randomly generated and located in the cell of radius of 150 meters. The distance of antenna elements are separated by half of a wavelength. Because of

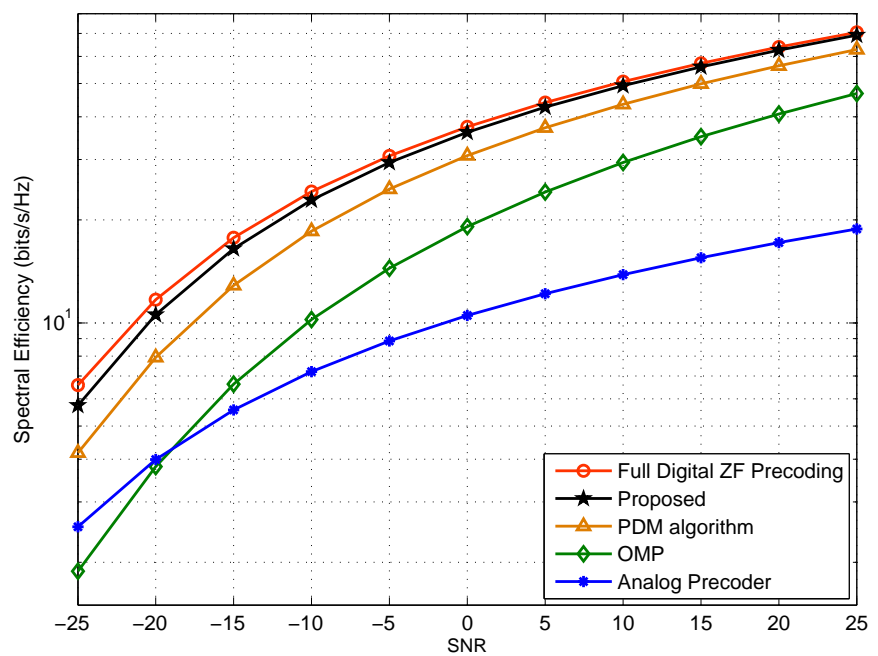

Fig. 5. Achievable SE comparison versus the SNR.

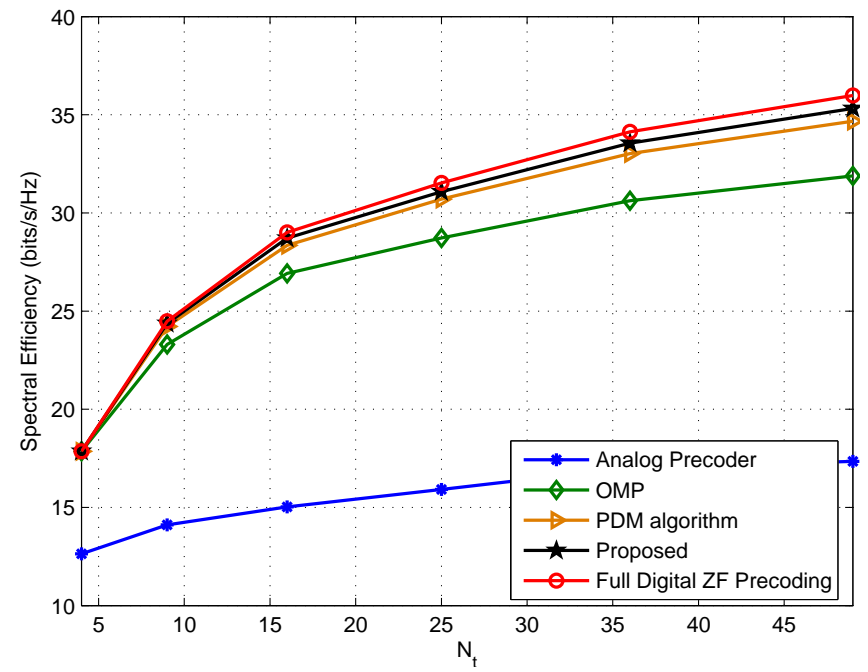

Fig. 6. Achievable SE comparison versus the number of antennas.

the constraint from the common law, usual practices and conventions, we set the minimum altitude of UAV $h_{\min }=20 \mathrm{~m}$ and the maximum altitude of UAV $h_{\max }=120 \mathrm{~m}$ respectively. All simulation results are done in a Monte Carlo fashion with 1000 realizations.

\section{A. Convergence Analysis}

The convergence of the proposed algorithm is investigated, as shown in Fig. 3 and 4. From the Fig. 3, it can be observed that after 80 iterations, the value of SE tends to be stable. From these results, we conclude that the proposed algorithm has a rapid convergence. In Fig. 4, we show the value of the constraint violation $\sum_{k=1}^{K}\left\|\boldsymbol{Q}_{k}-\overline{\boldsymbol{H}}_{k} \boldsymbol{W}\right\|_{F}^{2}$ versus the number of iterations for the proposed algorithm. It can be observed that the constraint violation reduces to a threshold $\epsilon=10^{-4}$, which indicates that the optimal solution has essentially satisfied with the equality constraints (20c) of the optimization problem (20). 


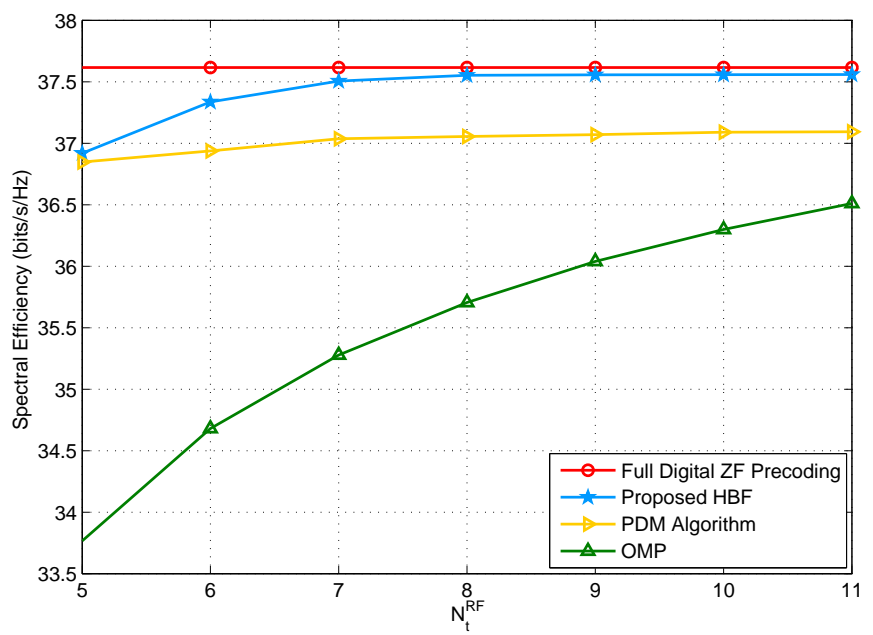

Fig. 7. Achievable SE versus the number of RF chains $(\mathrm{SNR}=10 \mathrm{~dB})$.

\section{B. Spectral Efficiency Evaluation}

Next, the SE achieved by all competing algorithms are investigated, where the number of RF chains and the data streams are set to the same. This is the worst case, since the number of RF chains cannot be smaller than data streams $N_{s}$, which also satisfies the constraints in Section II. For comparison, the performance of the full-digital ZF precoding scheme acts as a comparison benchmark precoding scheme (labeled by "FD-ZF"), and we compare the proposed scheme with the PDM [24] and OMP algorithm [49]. As shown in Fig. 5, the existing OMP algorithm achieves a very low SE in all competing methods. On the contrary, the SE of the proposed scheme is quite close to that of the fully digital ZF precoding scheme. This implies that an effective result can be obtained by the proposed scheme to approximate the fully digital ZF precoding, even though the RF chains are limited. Furthermore, the performance of the analog precoding structures reveals that only using phase shifters will inevitably lead to some non-negligible performance loss.

In Fig. 6, we further analyze the achievable SE comparison for different numbers of antennas. It is shown that when the number of BS antennas $N_{t}$ grows large, the SE increases without limit, if perfect hardware is assumed while it appears ceilings in practice. It is worth mentioning that when the number of BS antennas is small, the advantage of the proposed scheme is not obvious. In particular, we can observe the OMP, PDM algorithm and the proposed method converge the same $\mathrm{SE}$ value with the number of BS antennas $N_{t}=4$.

To verify the low hardware cost of the proposed method, the achievable SE of system versus the number of RF chains $N_{t}^{R F}$ has been shown in Fig. 7, where the number of RF chains $N_{t}^{R F}$ increases from 5 to 11 . It is observed that the performance of all the schemes in general improves with the increase of available RF chains. Interestingly, when the number of $N_{t}^{R F}$ exceeds 8 , the improvement of the proposed scheme is trivial. It is interesting that for all the schemes under consideration, the proposed scheme is equiped with only $6 \mathrm{RF}$ chains to achieve over 95 percent of SE in the full digital ZF precoding structure, but others competing methods need more ones.

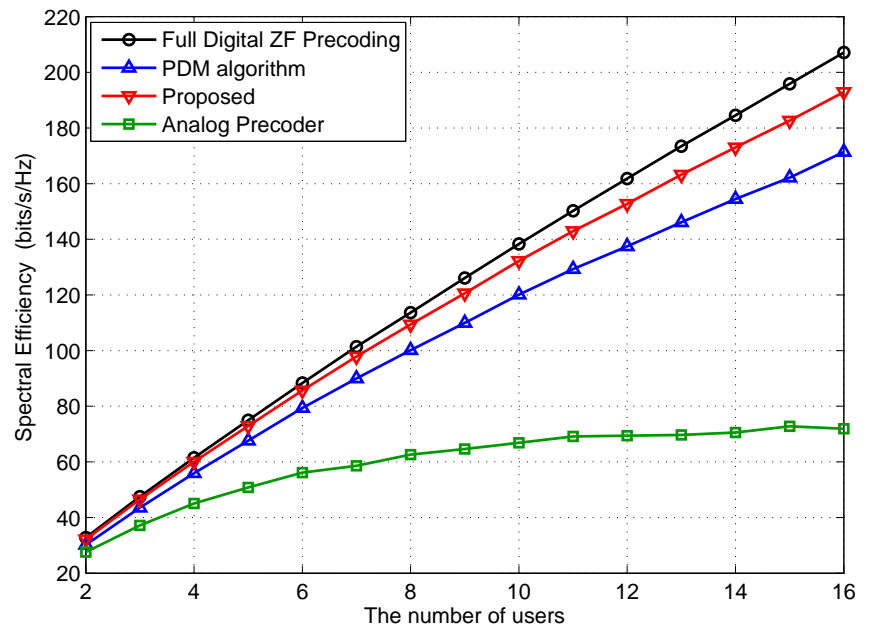

Fig. 8. Achievable SE comparison versus the number of GTs
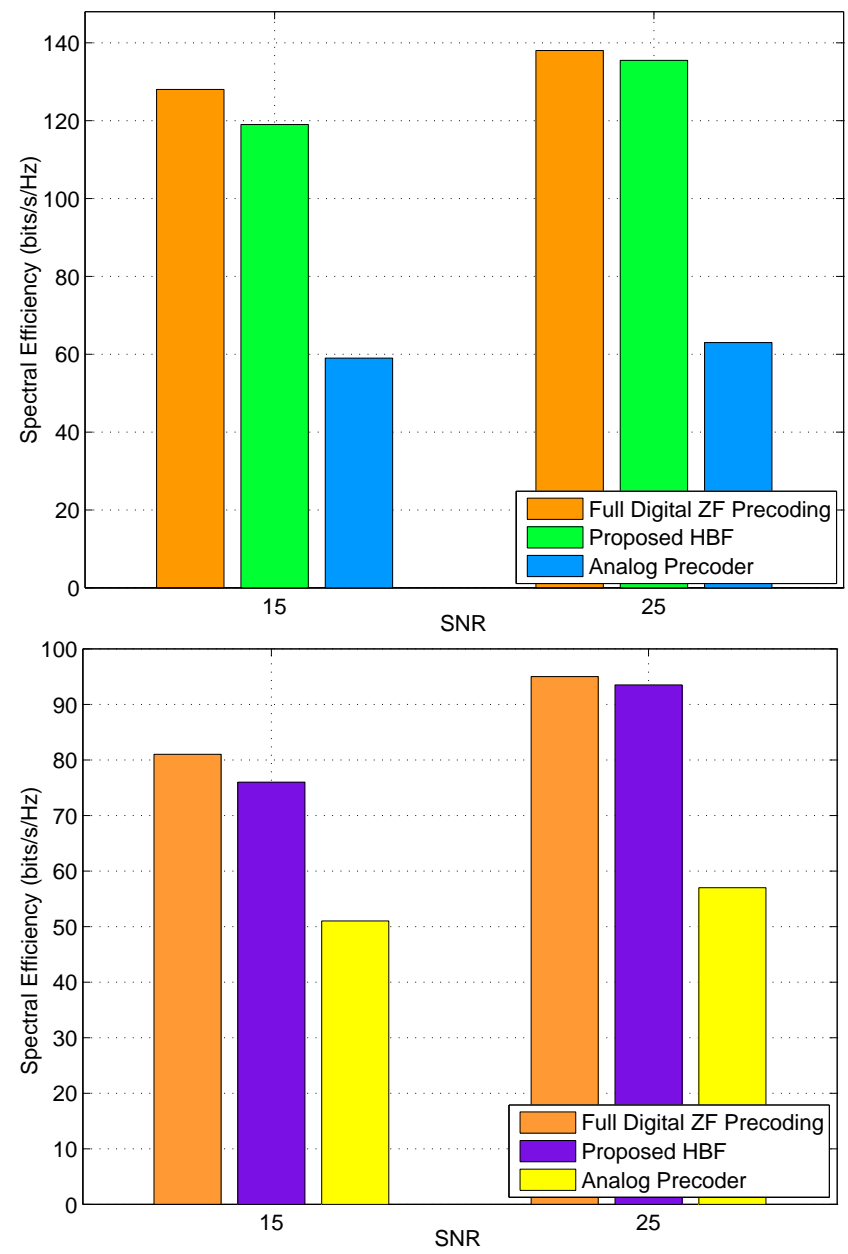

Fig. 9. SE comparison of the analog precoder, fully digital ZF precoder and FD-ZF Precoding (Above: $K=10$, Below: $K=6$ ).

\section{The Performance Evaluation of Different GTs}

Finally, we analyze the the achievable SE for the aforementioned schemes versus different numbers of GTs, where the number of GT increases from 2 to 16. In Fig. 8, it can be observed that except the analog precoding scheme, all the 


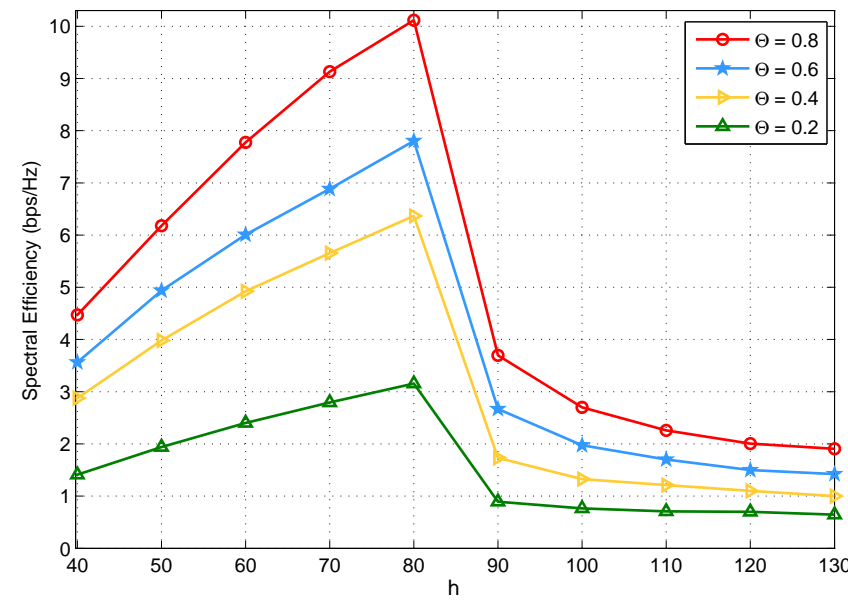

Fig. 10. Achievable SE comparison versus the altitude of UAV $h$.

other algorithms perform very close to each other under the small number of GTs. The performance of all these schemes increases monotonically with the number of GTs. Besides, the performance gap between the proposed scheme and PDM algorithm would escalate with the increasing number of GTs, which indicates that the proposed scheme has strong potential for large number of GTs scenario.

Fig. 9 illustrates the achievable spectrum efficiency of two existing precoding schemes and the proposed precoding scheme, respectively. It shows that our proposed scheme with LAAs achieves a significant improvement than the conventional analog precoding scheme. Compared to the full digital ZF precoding approach, the proposed precoders have tolerable system performance loss. However, the full digital ZF precoding scheme need more RF chains cost, which is not suitable for compact and lightweight design in UAV-enabled beamspace mmWave MIMO system.

\section{The Altitude Optimization of UAV}

To verify the necessity of altitude optimization of UAV, we refer the 3GPP specification [50], and the path loss is randomly determined by LoS and NLoS links according to probabilities. Specifically, the probability of the LOS is assumed as (36) and the the probability of the NLOS link is given by $P_{N L O S}=1-$ $P_{\text {LOS }}$. The achievable SE of the proposed scheme versus UAV altitude $h$ is illustrated in Fig. 10, where the $h$ value varies from 40 to $130 \mathrm{~m}$ and other parameters are kept unchanged. It can be seen that the achievable SE increases first and then decreases as $h$ increases. This is because that the probability of LoS link between the UAV and GT increases as the UAV height increasing. Therefore, the performance can be improved by increasing the UAV flight altitude within a certain range. However, when the altitude $h$ is sufficiently large, the distance between the UAV and the GT is increasing significantly, which results in high propagation path loss. Therefore, the altitude of UAV needs to be determined reasonably and carefully. To verify this issue, the altitude of UAV versus the number of RF chains/SNR/Users are provided in Fig. 11. The results show that the altitude of UAV $h$ reduces when the number of RF chains/users or SNR value are higher than a threshold value.
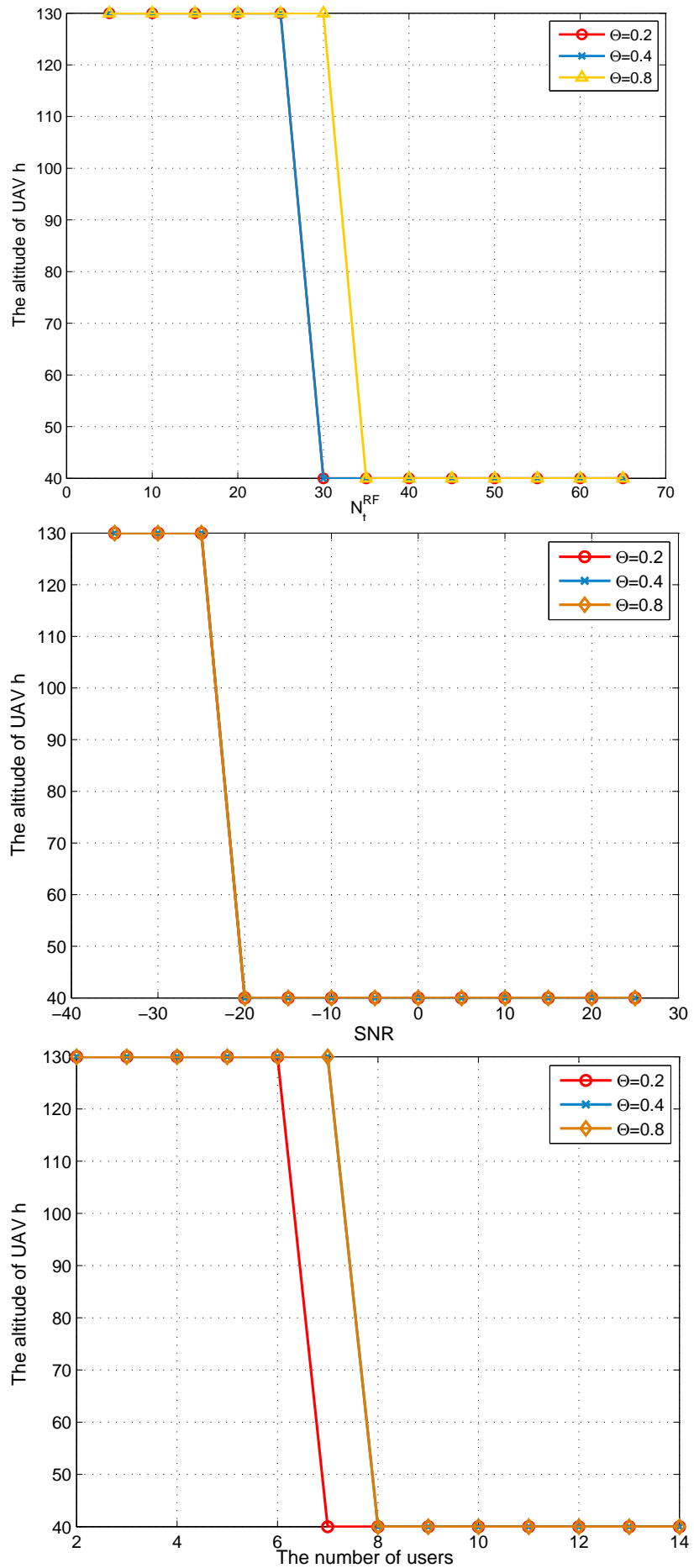

Fig. 11. The altitude of UAV $h$ comparison versus the number of RF chains $N_{t}^{R F}$ /SNR/users.

\section{CONCLUSION}

In this paper, the UAV with LAAs is considered to support multi-user transmission in UAV-enabled mmWave beamspace MIMO system. In particular, we focus on jointly optimizing altitude of UAV, transmit precoder, receive combiners as well as beam selector for the sum-rate maximization problem. The original problem is a non-convex, and transmit and receive variables are coupled with each other, which results 


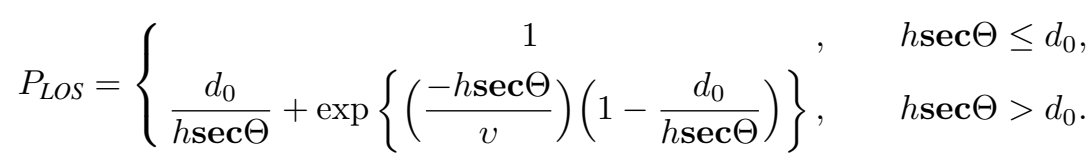

in extremely strenuous to solve the proposed optimization problem. To solve this limitation, the equivalent optimization problem with the weighted MSE criterion is developed to solve the original non-convex problem. To tackle the coupling constraints problem, an efficient algorithm is proposed based on the principle of alternating optimization, which transforms the original coupling constraints problem into a series of subproblems with separable constraints. Simulation results demonstrate that the proposed algorithm can achieve satisfactory performance and could converge in a few iterations.

\section{APPENDIX A}

THE PROOF OF THE PROBLEM (10)

Proof: Let $\overline{\boldsymbol{H}}_{k}=(h \mathbf{s e c} \Theta)^{-\alpha} \boldsymbol{H}_{k} \boldsymbol{S}$ for simplicity. The optimization problem (10) can be rewritten as

$$
\begin{aligned}
& \min _{\boldsymbol{W}, \boldsymbol{V}_{k}, \boldsymbol{S}, \xi_{k}, h} \sum_{k=1}^{K}\left(\log \operatorname{det}\left(\xi_{k}\right)-\operatorname{tr}\left(\xi_{k} \boldsymbol{E}_{k}\right)+N_{s}\right) \\
& \text { s.t. } \sum_{i=1}^{N_{t}} s_{i, j}=1, \quad \sum_{j=1}^{N_{r}^{R F}} s_{i, j} \leq 1, \quad s_{i, j} \in\{0,1\}, \\
& h_{\min } \leq h \leq h_{\max },
\end{aligned}
$$

where

$$
\boldsymbol{E}_{k}=\left(\boldsymbol{I}_{N_{r}}-\boldsymbol{V}_{k}^{H} \overline{\boldsymbol{H}} \boldsymbol{W}\right)\left(\boldsymbol{I}_{N_{r}}-\boldsymbol{V}_{k}^{H} \overline{\boldsymbol{H}} \boldsymbol{W}\right)^{H}+\sigma^{2} \boldsymbol{V}_{k}^{H} \boldsymbol{V}_{k} .
$$

Let $f\left(\xi_{k}\right)=\log \operatorname{det}\left(\xi_{k}\right)-\operatorname{tr}\left(\xi_{k} \boldsymbol{E}_{k}\right)+N_{s}$ for simplicity. The optimal $\xi_{k}$ is updated by taking the derivative of $f\left(\xi_{k}\right)$, we have

$$
\frac{\partial\left(\log \operatorname{det}\left(\xi_{k}\right)-\operatorname{tr}\left(\xi_{k} \boldsymbol{E}_{k}\right)\right)}{\partial \xi_{k}}=0 .
$$

After that, we can obtain the optimal $\xi_{k}$ as

$$
\xi_{k}^{*}=\boldsymbol{E}_{k}^{-1} .
$$

Similarly, considering $\overline{\boldsymbol{H}}_{k}=(h \mathbf{s e c} \Theta)^{-\alpha} \boldsymbol{H}_{k} \boldsymbol{S}$ in (37d), the combining matrix $\boldsymbol{V}_{k}$ can be rewritten as

$$
\boldsymbol{V}_{k}=\left(\overline{\boldsymbol{H}}_{k} \boldsymbol{W} \boldsymbol{W}^{H} \overline{\boldsymbol{H}}_{k}^{H}+\sigma^{2} \boldsymbol{I}_{N_{r}}\right)^{-1} \overline{\boldsymbol{H}}_{k} \boldsymbol{W} .
$$

Plugging $\boldsymbol{V}_{k}$ into (39) yields

$$
\xi_{k}^{*}=\left(\boldsymbol{I}_{N_{r}}-\boldsymbol{W}^{H} \overline{\boldsymbol{H}}_{k}^{H} \boldsymbol{J}_{k}^{-1} \overline{\boldsymbol{H}}_{k} \boldsymbol{W}\right)^{-1},
$$

where $\boldsymbol{J}_{k}=\sigma^{2} \boldsymbol{I}_{N_{r}}+\overline{\boldsymbol{H}}_{k} \boldsymbol{W} \boldsymbol{W}^{H} \overline{\boldsymbol{H}}_{k}^{H}$.

Applying the Woodbury matrix identity to $(41), f\left(\xi_{k}\right)$ is recast as

$$
\begin{aligned}
f\left(\xi_{k}\right) & =\log \operatorname{det}\left(\boldsymbol{E}_{k}^{-1}\right)-\operatorname{tr}\left(\xi_{k}^{*} \boldsymbol{E}_{k}\right)+N_{s} \\
& =\log \operatorname{det}\left(\boldsymbol{I}_{N_{r}}-\boldsymbol{W}^{H} \overline{\boldsymbol{H}}_{k}^{H} \boldsymbol{J}_{k}^{-1} \overline{\boldsymbol{H}}_{k} \boldsymbol{W}\right)^{-1} .
\end{aligned}
$$
lem

Thus, we have the following equivalent optimization prob-

$$
\begin{aligned}
\log \operatorname{det}\left(\xi_{k}\right) & =\log \operatorname{det}\left(\left(\boldsymbol{I}_{N_{r}}-\boldsymbol{W}^{H} \overline{\boldsymbol{H}}_{k}^{H} \boldsymbol{J}^{-1} \overline{\boldsymbol{H}}_{k} \boldsymbol{W}\right)^{-1}\right) \\
& \stackrel{(a)}{=} \log \operatorname{det}\left(\boldsymbol{I}_{N_{r}}+\sigma^{-2} \overline{\boldsymbol{H}}_{k} \boldsymbol{W} \boldsymbol{W}^{H} \overline{\boldsymbol{H}}_{k}^{H}\right) \\
& \stackrel{(b)}{=} \log \operatorname{det}\left(\boldsymbol{I}_{N_{r}}+\frac{\boldsymbol{H}_{k} \boldsymbol{S} \boldsymbol{W} \boldsymbol{W}^{H} \boldsymbol{S}^{H} \boldsymbol{H}_{k}^{H}}{\sigma^{2}(h \sec \Theta)^{2 \alpha}}\right)
\end{aligned}
$$

where $(a)$ is due to the identity $\operatorname{det}(\boldsymbol{I}+\boldsymbol{X} \boldsymbol{Y})=\operatorname{det}(\boldsymbol{I}+$ $\boldsymbol{Y} \boldsymbol{X})$ and Woodbury matrix identity; $(b)$ is from that $\overline{\boldsymbol{H}}_{k}=$ $(h \mathbf{s e c} \Theta)^{-\alpha} \boldsymbol{H}_{k} \boldsymbol{S}$. Therefore, the optimization problem (10) is equivalent to the optimization problem (8) and the proof is completed.

\section{ACKNOWLEDGMENT}

The authors wish to acknowledge Boyi Tang, University of Electronic Science and Technology of China, for assistance with the experimental design about the altitude of UAV v.s. $N_{t}^{R F} / \mathrm{SNR} /$ number of users.

\section{REFERENCES}

[1] Z. Yang, C. Pan, M. Shikh-Bahaei, W. Xu, M. Chen, M. Elkashlan, and A. Nallanathan, "Joint altitude, beamwidth, location, and bandwidth optimization for UAV-enabled communications," IEEE Communications Letters, vol. 22, no. 8, pp. 1716-1719, 2018.

[2] M. Liu, J. Yang, and G. Gui, "DSF-NOMA: UAV-assisted emergency communication technology in a heterogeneous internet of things," IEEE Internet of Things Journal, vol. 6, no. 3, pp. 5508-5519, 2019.

[3] W. Yi, Y. Liu, E. Bodanese, A. Nallanathan, and G. K. Karagiannidis, "A unified spatial framework for UAV-aided mmwave networks," IEEE Transactions on Communications, vol. 67, no. 12, pp. 8801-8817, 2019.

[4] Z. Xiao, P. Xia, and X. Xia, "Enabling UAV cellular with millimeterwave communication: potentials and approaches," IEEE Communications Magazine, vol. 54, no. 5, pp. 66-73, 2016.

[5] P. Yu, W. Li, F. Zhou, L. Feng, M. Yin, S. Guo, Z. Gao, and X. Qiu, "Capacity enhancement for $5 \mathrm{G}$ networks using mmwave aerial base stations: Self-organizing architecture and approach," IEEE Wireless Communications, vol. 25, no. 4, pp. 58-64, 2018.

[6] M. T. Dabiri, H. Safi, S. Parsaeefard, and W. Saad, "Analytical channel models for millimeter wave UAV networks under hovering fluctuations," IEEE Transactions on Wireless Communications, vol. 19, no. 4, pp. 2868-2883, 2020.

[7] P. V. Amadori and C. Masouros, "Low RF-complexity millimeter-wave beamspace-MIMO systems by beam selection," IEEE Transactions on Communications, vol. 63, no. 6, pp. 2212-2223, 2015.

[8] Y. Sun and C. Qi, "Weighted sum-rate maximization for analog beamforming and combining in millimeter wave massive MIMO communications," IEEE Communications Letters, vol. 21, no. 8, pp. 1883-1886, 2017.

[9] Shuguang Cui, A. J. Goldsmith, and A. Bahai, "Energy-efficiency of MIMO and cooperative MIMO techniques in sensor networks," IEEE Journal on Selected Areas in Communications, vol. 22, no. 6, pp. 10891098, 2004.

[10] Z. Chen, J. Tang, H. Tang, X. Zhang, D. K. C. So, and K.-K. Wong, "Channel estimation of IRS-aided communication systems with hybrid multiobjective optimization," in ICC 2021 - IEEE International Conference on Communications, pp. 1-6, 2021. 
[11] Z. Chen, J. Tang, X. Zhang, Q. Wu, Y. Wang, D. K. C. So, S. Jin, and K.-K. Wong, "Offset learning based channel estimation for intelligent reflecting surface-assisted indoor communication," IEEE Journal of Selected Topics in Signal Processing, pp. 1-14, 2021.

[12] M. Artuso, D. Boviz, A. Checko, H. L. Christiansen, B. Clerckx, L. Cottatellucci, D. Gesbert, B. Gizas, A. Gopalasingham, F. Khan, J. Kelif, R. Mller, D. Ntaikos, K. Ntougias, C. B. Papadias, B. Rassouli, M. A. Sedaghat, T. Ratnarajah, L. Roullet, S. Senecal, H. Yin, and L. Zhou, "Enhancing LTE with Cloud-RAN and load-controlled parasitic antenna arrays," IEEE Communications Magazine, vol. 54, no. 12, pp. 183-191, 2016.

[13] K. Ntougias, D. K. Ntaikos, C. B. Papadias, and G. K. Papageorgiou, "Coordinated hybrid precoding and QoS-aware power allocation for underlay spectrum sharing with load-controlled antenna arrays," in 2019 IEEE 20th International Workshop on Signal Processing Advances in Wireless Communications (SPAWC), pp. 1-5, 2019.

[14] B. H. Wang, H. T. Hui, and M. S. Leong, "Global and fast receiver antenna selection for MIMO systems," IEEE Transactions on Communications, vol. 58, no. 9, pp. 2505-2510, 2010.

[15] Y. Gao, H. Vinck, and T. Kaiser, "Massive MIMO antenna selection: Switching architectures, capacity bounds, and optimal antenna selection algorithms," IEEE Transactions on Signal Processing, vol. 66, no. 5, pp. 1346-1360, 2018.

[16] W. Shen, X. Bu, X. Gao, C. Xing, and L. Hanzo, "Beamspace precoding and beam selection for wideband millimeter-wave MIMO relying on lens antenna arrays," IEEE Transactions on Signal Processing, vol. 67 , no. 24, pp. 6301-6313, 2019.

[17] V. V. Ratnam, A. F. Molisch, O. Y. Bursalioglu, and H. C. Papadopoulos, "Hybrid beamforming with selection for multiuser massive MIMO systems," IEEE Transactions on Signal Processing, vol. 66, no. 15, pp. 4105-4120, 2018.

[18] M. R. Akdeniz, Y. Liu, M. K. Samimi, S. Sun, S. Rangan, T. S. Rappaport, and E. Erkip, "Millimeter wave channel modeling and cellular capacity evaluation," IEEE Journal on Selected Areas in Communications, vol. 32, no. 6, pp. 1164-1179, 2014.

[19] X. Gao, L. Dai, S. Han, C. I, and R. W. Heath, "Energy-efficient hybrid analog and digital precoding for mmwave MIMO systems with large antenna arrays," IEEE Journal on Selected Areas in Communications, vol. 34, no. 4, pp. 998-1009, 2016.

[20] J. Zhang, Y. Huang, J. Wang, and L. Yang, "Hybrid precoding for wideband millimeter-wave systems with finite resolution phase shifters," IEEE Transactions on Vehicular Technology, vol. 67, no. 11, pp. 1128511290, 2018.

[21] R. Guo, Y. Cai, M. Zhao, Q. Shi, B. Champagne, and L. Hanzo, "Joint design of beam selection and precoding matrices for mmwave MU-MIMO systems relying on lens antenna arrays," IEEE Journal of Selected Topics in Signal Processing, vol. 12, no. 2, pp. 313-325, 2018.

[22] R. Mndez-Rial, C. Rusu, N. Gonzlez-Prelcic, A. Alkhateeb, and R. W. Heath, "Hybrid MIMO architectures for millimeter wave communications: Phase shifters or switches?," IEEE Access, vol. 4, pp. 247-267, 2016.

[23] Y. Zeng, R. Zhang, and Z. N. Chen, "Electromagnetic lens-focusing antenna enabled massive MIMO: Performance improvement and cost reduction," IEEE Journal on Selected Areas in Communications, vol. 32, no. 6, pp. 1194-1206, 2014.

[24] Y. Zeng and R. Zhang, "Millimeter wave MIMO with lens antenna array: A new path division multiplexing paradigm," IEEE Transactions on Communications, vol. 64, no. 4, pp. 1557-1571, 2016.

[25] T. Xie, L. Dai, D. W. K. Ng, and C. Chae, "On the power leakage problem in millimeter-wave massive MIMO with lens antenna arrays," IEEE Transactions on Signal Processing, vol. 67, no. 18, pp. 4730-4744, 2019.

[26] W. Shen, L. Dai, Y. Li, Z. Wang, and L. Hanzo, "Channel feedback codebook design for millimeter-wave massive MIMO systems relying on lens antenna array," IEEE Wireless Communications Letters, vol. 7, no. 5, pp. 736-739, 2018.

[27] C. Feng, W. Shen, and J. An, "Beam selection for wideband millimeter wave MIMO relying on lens antenna arrays," IEEE Communications Letters, vol. 23, no. 10, pp. 1875-1878, 2019.

[28] X. Yu, J. Zhang, and K. B. Letaief, "A hardware-efficient analog network structure for hybrid precoding in millimeter wave systems," IEEE Journal of Selected Topics in Signal Processing, vol. 12, no. 2, pp. 282-297, 2018.

[29] X. Gao, L. Dai, Z. Chen, Z. Wang, and Z. Zhang, "Near-optimal beam selection for beamspace mmwave massive MIMO systems," IEEE Communications Letters, vol. 20, no. 5, pp. 1054-1057, 2016.
[30] W. Feng, J. Wang, Y. Chen, X. Wang, N. Ge, and J. Lu, "UAV-aided MIMO communications for $5 \mathrm{G}$ internet of things," IEEE Internet of Things Journal, vol. 6, no. 2, pp. 1731-1740, 2019.

[31] M. Mozaffari, A. Taleb Zadeh Kasgari, W. Saad, M. Bennis, and M. Debbah, "Beyond 5G with UAVs: Foundations of a 3D wireless cellular network," IEEE Transactions on Wireless Communications, vol. 18, no. 1, pp. 357-372, 2019.

[32] H. Huang, Y. Yang, H. Wang, Z. Ding, H. Sari, and F. Adachi, "Deep reinforcement learning for UAV navigation through massive MIMO technique," IEEE Transactions on Vehicular Technology, vol. 69, no. 1, pp. 1117-1121, 2020 .

[33] C. Liu, K. Ho, and J. Wu, "Mmwave UAV networks with multi-cell association: Performance limit and optimization," IEEE Journal on Selected Areas in Communications, vol. 37, no. 12, pp. 2814-2831, 2019.

[34] J. Du, W. Xu, Y. Deng, A. Nallanathan, and L. Vandendorpe, "Energysaving UAV-assisted multiuser communications with massive MIMO hybrid beamforming," IEEE Communications Letters, vol. 24, no. 5, pp. 1100-1104, 2020.

[35] A. Alkhateeb and R. W. Heath, "Frequency selective hybrid precoding for limited feedback millimeter wave systems," IEEE Transactions on Communications, vol. 64, no. 5, pp. 1801-1818, 2016.

[36] Y. Zeng, L. Yang, and R. Zhang, "Multi-user millimeter wave MIMO with full-dimensional lens antenna array," IEEE Transactions on Wireless Communications, vol. 17, no. 4, pp. 2800-2814, 2018.

[37] J. Brady, N. Behdad, and A. M. Sayeed, "Beamspace MIMO for millimeter-wave communications: System architecture, modeling, analysis, and measurements," IEEE Transactions on Antennas and Propagation, vol. 61, no. 7, pp. 3814-3827, 2013

[38] A. Sayeed and J. Brady, "Beamspace MIMO for high-dimensional multiuser communication at millimeter-wave frequencies," in 2013 IEEE Global Communications Conference (GLOBECOM), pp. 3679-3684, 2013.

[39] X. Gao, L. Dai, S. Han, C. I, and X. Wang, "Reliable beamspace channel estimation for millimeter-wave massive MIMO systems with lens antenna array," IEEE Transactions on Wireless Communications, vol. 16, no. 9, pp. 6010-6021, 2017.

[40] H. Li, M. Li, Q. Liu, and A. L. Swindlehurst, "Dynamic hybrid beamforming with low-resolution PSs for wideband mmwave MIMOOFDM systems," IEEE Journal on Selected Areas in Communications, vol. 38, no. 9, pp. 2168-2181, 2020 .

[41] Z. Chen, J. Tang, X. Y. Zhang, D. K. C. So, S. Jin, and K.-K. Wong, "Hybrid evolutionary-based sparse channel estimation for irsassisted mmwave MIMO systems," IEEE Transactions on Wireless Communications, pp. 1-13, 2021.

[42] L. Liu, S. Zhang, and R. Zhang, "Multi-beam UAV communication in cellular uplink: Cooperative interference cancellation and sum-rate maximization," IEEE Transactions on Wireless Communications, vol. 18, no. 10, pp. 4679-4691, 2019

[43] B. Wang, L. Dai, Z. Wang, N. Ge, and S. Zhou, "Spectrum and energyefficient beamspace MIMO-NOMA for millimeter-wave communications using lens antenna array," IEEE Journal on Selected Areas in Communications, vol. 35, no. 10, pp. 2370-2382, 2017.

[44] J. Joung and Y. H. Lee, "Regularized channel diagonalization for multiuser MIMO downlink using a modified MMSE criterion," IEEE Transactions on Signal Processing, vol. 55, no. 4, pp. 1573-1579, 2007.

[45] S. Mertens, "Exhaustive search for low-autocorrelation binary sequences," Journal of Physics A: Mathematical and General, vol. 29, no. 18 , pp. $54-73,1996$.

[46] Q. Shi, M. Razaviyayn, Z. Luo, and C. He, "An iteratively weighted MMSE approach to distributed sum-utility maximization for a MIMO interfering broadcast channel," IEEE Transactions on Signal Processing, vol. 59, no. 9, pp. 4331-4340, 2011.

[47] Q. Shi and M. Hong, "Penalty dual decomposition method with application in signal processing," in 2017 IEEE International Conference on Acoustics, Speech and Signal Processing (ICASSP), pp. 4059-4063, 2017.

[48] Q. Shi and M. Hong, "Penalty dual decomposition method for nonsmooth nonconvex optimization-part I: Algorithms and convergence analysis," IEEE Transactions on Signal Processing, vol. 68, pp. 41084122,2020

[49] O. E. Ayach, S. Rajagopal, S. Abu-Surra, Z. Pi, and R. W. Heath, "Spatially sparse precoding in millimeter wave MIMO systems," IEEE Transactions on Wireless Communications, vol. 13, no. 3, pp. 14991513, 2014.

[50] 3GPP, “3GPP TR 36.777," in Study on Enhanced LTE Support for Aerial Vehicles (Release 15), Dec. 2017. 